\title{
Physiological and Proteomic Responses of Diploid and Tetraploid Black Locust (Robinia pseudoacacia L.) Subjected to Salt Stress
}

\section{Zhiming Wang ${ }^{1, \dagger}$, Mingyue Wang ${ }^{1, \dagger}$, Likun Liu ${ }^{2}$ and Fanjuan Meng ${ }^{1, *}$}

1 College of Life Science, Northeast Forestry University, Harbin 150040, China; E-Mails: 13674678261@163.com (Z.W.); 13633662163@163.com (M.W.)

2 Department of Medical Biotechnology, College of Biomedical Science, Kangwon National University, Chuncheon, Gangwon-do 200-701, Korea; E-Mail: 1lk_2008@126.com

$\dagger$ These authors contributed equally to this work.

* Author to whom correspondence should be addressed; E-Mail: mfj19751@163.com; Tel.: +86-451-8219-2170; Fax: +86-451-8643-3905.

Received: 17 June 2013; in revised form: 31 August 2013 / Accepted: 9 September 2013 / Published: 14 October 2013

\begin{abstract}
Tetraploid black locust (Robinia pseudoacacia L.) is adaptable to salt stress. Here, we compared morphological, physiological, ultrastructural, and proteomic traits of leaves in tetraploid black locust and its diploid relatives under salt stress. The results showed that diploid $(2 \times)$ plants suffered from greater negative effects than those of tetraploid $(4 \times)$ plants. After salt treatment, plant growth was inhibited, photosynthesis was reduced, reactive oxygen species, malondialdehyde content, and relative electrolyte leakage increased, and defense-related enzyme activities decreased in $2 \times$ compared to those in $4 \times$. In addition, salt stress resulted in distorted chloroplasts, swollen thylakoid membranes, accumulation of plastoglobules, and increased starch grains in $2 \times$ compared to those in $4 \times$. However, $4 \times$ developed diverse responses under salt stress. A comparative proteomic analysis revealed that 41 and 37 proteins were differentially expressed in $2 \times$ and $4 \times$, respectively. These proteins were mainly involved in photosynthesis, stress and defense, energy, metabolism, transcription/translation, and transportation. Distinct patterns of protein changes between $2 \times$ and $4 \times$ were analyzed. Collectively, our results suggest that the plants showed significantly different responses to salt stress based on ploidy level of
\end{abstract}


the plant. The $4 \times$ possessed a better salt protection mechanism than that of $2 \times$, suggesting salt tolerance in the polyploid plant.

Keywords: salt stress; Robinia pseudoacacia L.; diploid; tetraploid; physiology; proteomics

Abbreviations: APX, ascorbate peroxidase; BGR, the relative growth rate of stem basal diameter; BSA, bovine serum albumin; CHS, chalcone synthase; 2-DE, Two-dimensional electrophoresis; HGR, The relative growth rate of height; $\mathrm{H}_{2} \mathrm{O}_{2}$, hydrogen peroxide; Hsp70, heat shock protein 70; MDA, Malondialdehyde; PGK, phosphoglycerate kinase; PPK, phosphoribulokinase; RGR, the relative growth rate of range; ROS, Reactive Oxygen Species; RuBPCase, ribulose-bisphosphate carboxylase; RWC, relative water content; SBPase, sedoheptulose-1,7-bisphosphatase; TCA, tricarboxylic acid.

\section{Introduction}

Soil salinity is a major environmental stressor that severely limits crop growth and harvest worldwide [1]. In general, salinity stress induces deleterious cellular changes, including water deficits, ion homeostasis, ionic toxicity, membrane alterations, and free radical production [2,3]. Many plants grow slowly or die under salt stress. Plants have evolved complex mechanisms to adapt to salt stress based on modifications in metabolites, gene expression and proteins. To date, many genes responding to salt stress in plants have been identified [4]. However, these genes do not offer insight into the quantity and quality of the final gene products, i.e., the proteins that are regulated at the translational level [5]. Proteomics is a necessary and crucial component to genomic approaches and a powerful tool that facilitates the analysis of biochemical pathways and the complex response mechanisms of plants to the stress caused by salt, cold, and drought [6,7]. Earlier reports based on proteomics have mainly focused on the responses of diploid $(2 \times)$ plants, and the proteomic knowledge of the response to salt stress by polyploids is very limited.

Polyploidy arises from the doubling of chromosomes of a single species (autopolyploidy) or the hybrids between two species (allopolyploidy). Polyploidy usually changes anatomical and morphological characteristics such as an increase in leaf thickness and pubescence [8,9]. Moreover, polyploidy also changes physiological functions or gene expression [10-12]. Accordingly, these changes may affect a lot the phenotype and the response to stress [13-18]. Due to the interest in a homogenous genetic background, some studies have tried to compare polyploids and their diploid relatives for tolerance to environmental stressors. Polyploids clearly exhibit higher tolerance to drought, heat, cold, salt, viruses, fungi, and pest stressors compared with their diploid relatives [19-22]. Therefore, polyploidy has been regarded as an efficient way to improve environmental stress tolerance in plants and has played important roles in agriculture and forestry [23]. However, the molecular and physiological basis of stress tolerance by polyploids is not well understood.

Tetraploid (4×) black locust (Robinia pseudoacacia L.), which is native to Korea, is a preferred tree species in the timber forest due to its rapid growth and good wood texture. Moreover, it can be used as fine feed for domestic fowl and livestock because its fleshy leaves are rich in vitamins and minerals. 
Importantly, tetraploid black locust is a pioneer tree species due to it wide ranging adaptability to adverse environments such as salt, drought, cold, and pests. Accordingly, tetraploid black locust has high ecological and economic value.

In this study, we report on the physiological and proteomic responses to salt stress in $2 \times$ and $4 \times$ black locust. The objectives of this study were (1) to determine growth and physiological traits under salt stress in $2 \times$ and $4 \times$ black locust (2) to improve the understanding of the molecular mechanism of differential salt tolerance in different ploidy species, and (3) to aid in the rational engineering of plants with enhanced salt tolerance.

\section{Results and Discussion}

\subsection{Plant Growth and Physiological Response of Leaves of $2 \times$ and $4 \times$ Plants to Salt Stress}

Diploid plants began to wilt after 10 days and some plants died after 15 days under salt stress, as shown in Figure 1. However, $4 \times$ plants did not show significant changes even at the end of the 15 days experiment (Figure 1). In addition, salt treatment significantly inhibited plant growth (relative height growth rate (HGR) and the relative stem basal diameter growth rate (BGR)), but the difference in relative growth rate $(\mathrm{RGR})$ was not significant between $2 \times$ and $4 \times$ (Figure $2 \mathrm{~A}-\mathrm{C}$ ). In contrast, after a 10 days salt treatment, relative water content (RWC) decreased in both $2 \times$ and $4 \times$, whereas RWC in $2 \times$ was much higher than that in $4 \times$ after 10 days of salt stress (Figure 2D).

Figure 1. Morphological changes in Robinia pseudoacacia diploid $(2 \times)$ and tetraploid $(4 \times)$ plants growth after salt treatment $(500 \mathrm{mM} \mathrm{NaCl})$. Diploid (A) and tetraploid (4×) (B) plants were grown in a soil and sand mixture (2:1) after 1, 5, 10, and 15 days of salt treatment.

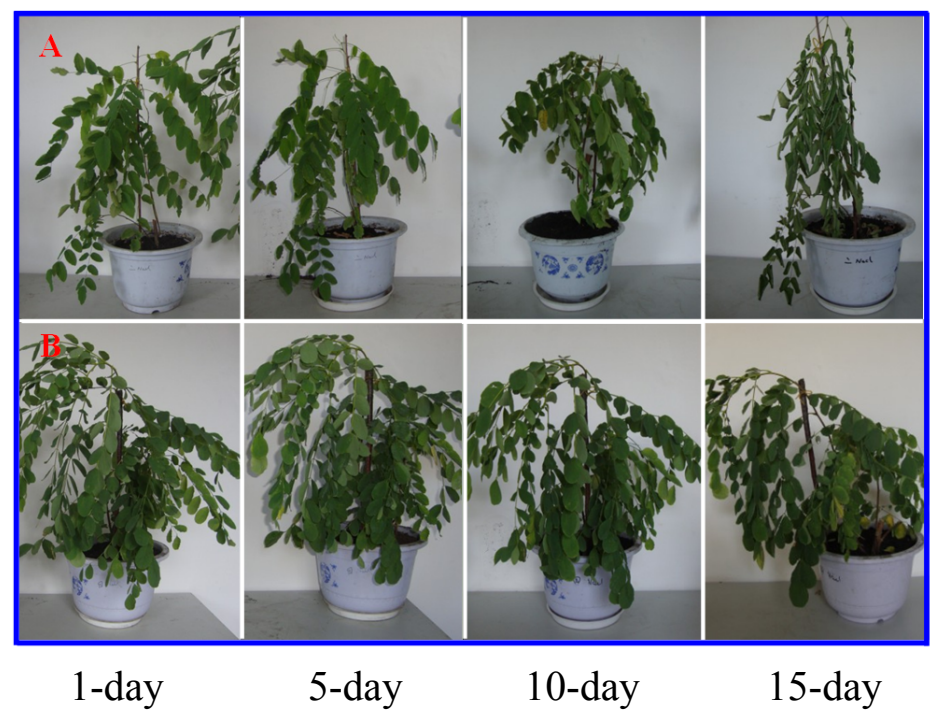

Salinity increased $\mathrm{O}_{2}^{-}$and $\mathrm{H}_{2} \mathrm{O}_{2}$ contents in both $2 \times$ and $4 \times$ plants (Figure $3 \mathrm{~A}, \mathrm{~B}$ ). However, $2 \times$ showed higher $\mathrm{O}_{2}^{-}$and $\mathrm{H}_{2} \mathrm{O}_{2}$ contents than did $4 \times$ at the end of 10 days of the experiment. Additionally, malondialdehyde (MDA) content and relative electrolyte leakage (REL) increased in $2 \times$ and $4 \times$ after salt treatment, and $2 \times$ showed much higher levels than that of $4 \times($ Figure $3 \mathrm{C}, \mathrm{D})$. 
Figure 2. Effects of salt stress on relative height growth rate (HGR) (A), relative range growth rate (RGR) (B), relative basal diameter growth rate (BGR) $(\mathbf{C})$, and relative water content (RWC) (D) of leaves in Robinia pseudoacacia diploid (2×) (black bars) and tetraploid $(4 \times)$ (white bars) plants growing under salt stress. Values followed by different letters are significantly different from each other at $p<0.05$ according to Duncan's method. Each data point represents the mean \pm standard error of three replicates.

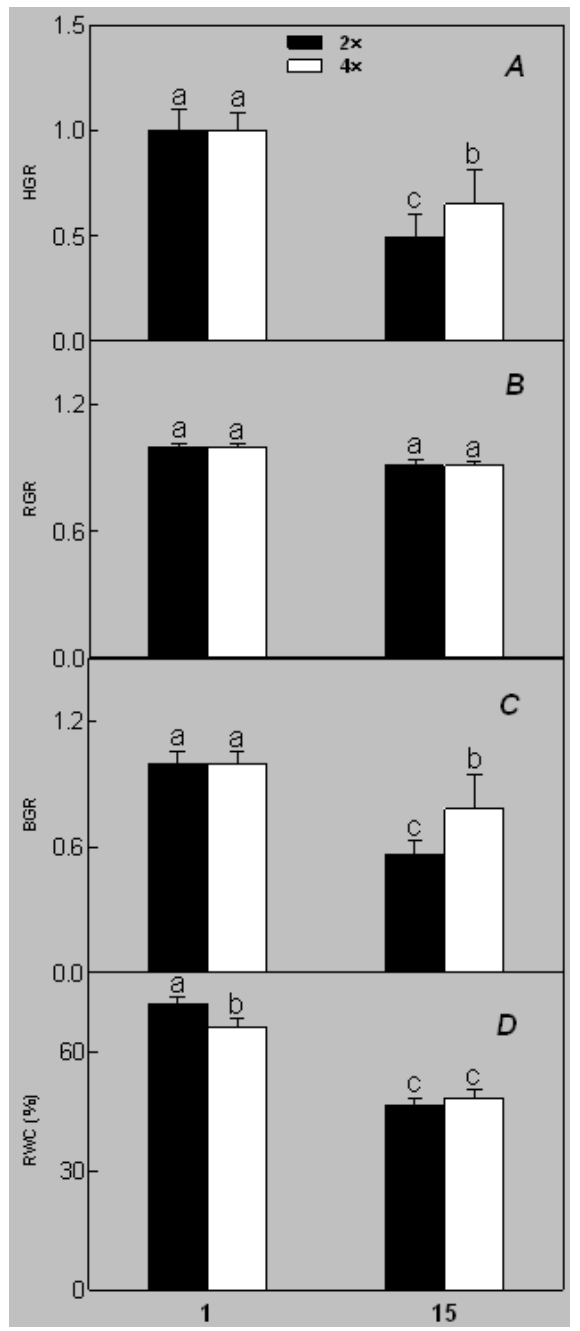

At day 1 after the $\mathrm{NaCl}$ treatment, concentrations of $\mathrm{Cl}^{-}, \mathrm{K}^{+}$, and $\mathrm{Na}^{+}$were higher in $4 \times$ than those in $2 \times$ plants. The $\mathrm{K}^{+} / \mathrm{Na}^{+}$ratios were similar in both $2 \times$ and $4 \times$ (Figure $4 \mathrm{~A}-\mathrm{D}$ ). After salt treatment, $\mathrm{Na}^{+}$, and $\mathrm{Cl}^{-}$increased in $2 \times$; however, no significant changes were detected in $4 \times$ (Figure 4A,B). In addition, $\mathrm{NaCl}$ treatment caused no significant changes in $\mathrm{K}^{+}$concentration in $2 \times$. However, it only caused a slight increase in the $\mathrm{K}^{+}$level in $4 \times$ (Figure $4 \mathrm{C}$ ). The $\mathrm{K}^{+} / \mathrm{Na}^{+}$ratio exhibited a significant decrease in $2 \times$ when exposed to salinity but they changed little in $4 \times$ (Figure 4D). 
Figure 3. Effects of salt stress on $\mathrm{O}_{2}^{-}$content $(\mathbf{A}), \mathrm{H}_{2} \mathrm{O}_{2}$ content $(\mathbf{B})$, and malondialdehyde (MDA) content (C) and relative electrolyte leakage (REL) (D) in the leaves of Robinia pseudoacacia diploid $(2 \times)$ (black bars) and tetraploid $(4 \times)$ (white bars) plants growing under salt stress. Values followed by different letters are significantly different from each other at $p<0.05$ according to Duncan's method. Each data point represents the mean \pm standard error of three replicates.

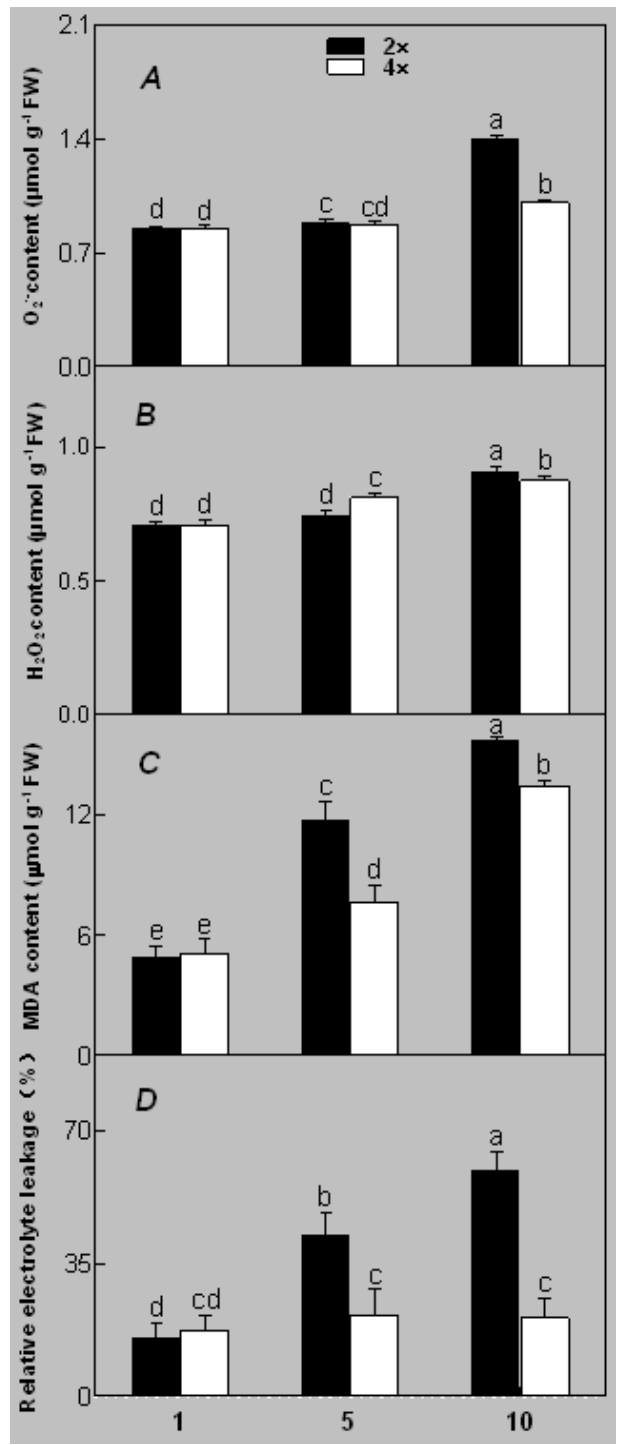

During different treatments, net $\mathrm{CO}_{2}$ assimilation rate $(P n)$ and stomatal conductance $(G s)$ were higher in $4 \times$ than those in $2 \times$ (Figure 5A,B). In $2 \times, P n$ and $G s$ declined when plants were exposed to salinity. However, $P n$ and $G s$ in $4 \times$ plants were not significantly affected by salinity. Additionally, intercellular $\mathrm{CO}_{2}$ concentration $(\mathrm{Ci})$ of $2 \times$ and $4 \times$ did not decrease significantly under salt treatment (Figure 5C). 
Figure 4. Effects of salt stress on the concentrations of $\mathrm{Na}^{+}(\mathbf{A}), \mathrm{Cl}^{-}(\mathbf{B}), \mathrm{K}^{+}(\mathbf{C})$, and the $\mathrm{K}^{+} / \mathrm{Na}^{+}$ratio (D) in the leaves of Robinia pseudoacacia diploid (2×) (black bars) and tetraploid $(4 \times)$ (white bars) plants growing after salt treatment. Values followed by different letters are significantly different from each other at $p<0.05$ according to Duncan's method. Each data point represents the mean \pm standard error of three replicates.

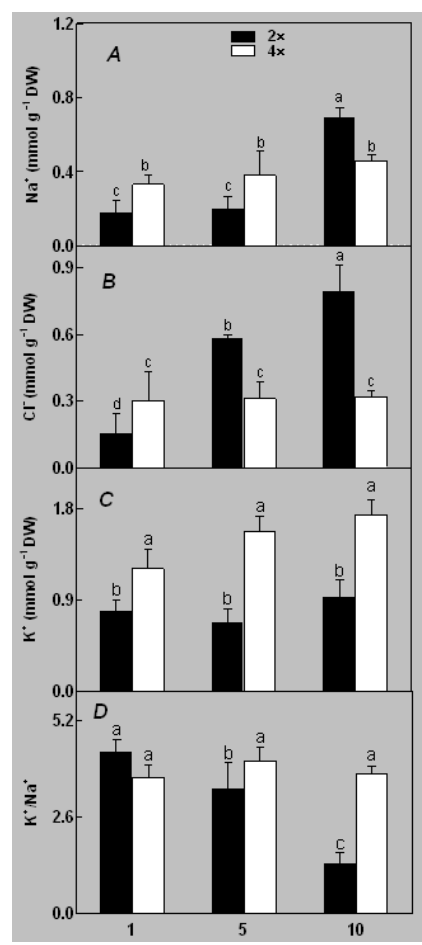

Figure 5. Effects of salt stress on net $\mathrm{CO}_{2}$ assimilation rate $(\mathrm{Pn})(\mathbf{A})$, stomatal conductance $(\mathrm{Gs})(\mathbf{B})$, and intercellular $\mathrm{CO}_{2}$ concentration $(\mathrm{Ci})(\mathbf{C})$ in the leaves of Robinia pseudoacacia diploid $(2 \times)$ (black bars) and tetraploid $(4 \times)$ (white bars) plants growing under salt stress. Values followed by different letters are significantly different from each other at $p<0.05$ according to Duncan's method. Each data point represents the mean \pm standard error of three replicates.

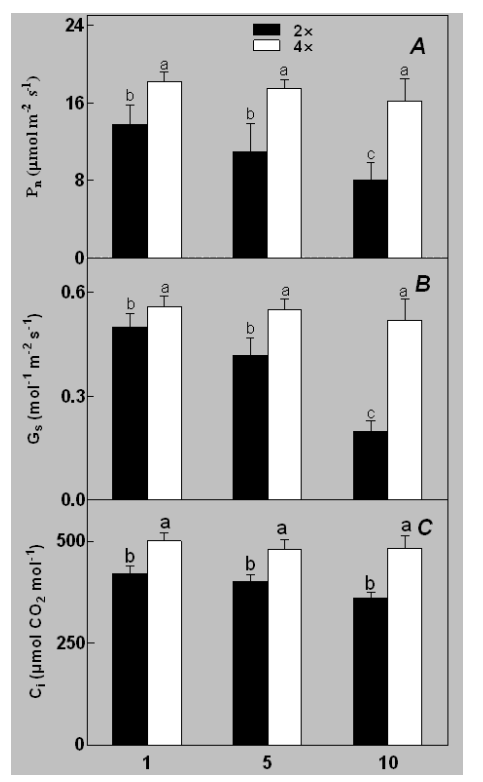


Superoxide dismutase (SOD) activity decreased in $2 \times$ and $4 \times$ after salt treatment, but $4 \times$ showed higher SOD activity than that of $2 \times$ (Figure 6A). The activities of peroxidase (POD), ascorbate peroxidase (APX), and glutathione reductase (GR) increased under salt stress in $4 \times$ but not in $2 \times$ (Figure 6B-D). In particular, $4 \times$ plants showed significantly higher APX and GR activities than those of $2 \times$ when subjected to salinity (Figure $6 \mathrm{C}, \mathrm{D}$ ).

Figure 6. Effects of salt stress on the activities of superoxide dismutase (SOD) (A), peroxidase (POD) (B), ascorbate peroxidase (APX) (C), and glutathione reductase (GR) (D) in the leaves of Robinia pseudoacacia diploid $(2 \times)$ (black bars) and tetraploid $(4 \times)$ (white bars) plants growing after salt treatment. Values followed by different letters are significantly different from each other at $p<0.05$ according to Duncan's method. Each data point represents the mean \pm standard error of three replicates.

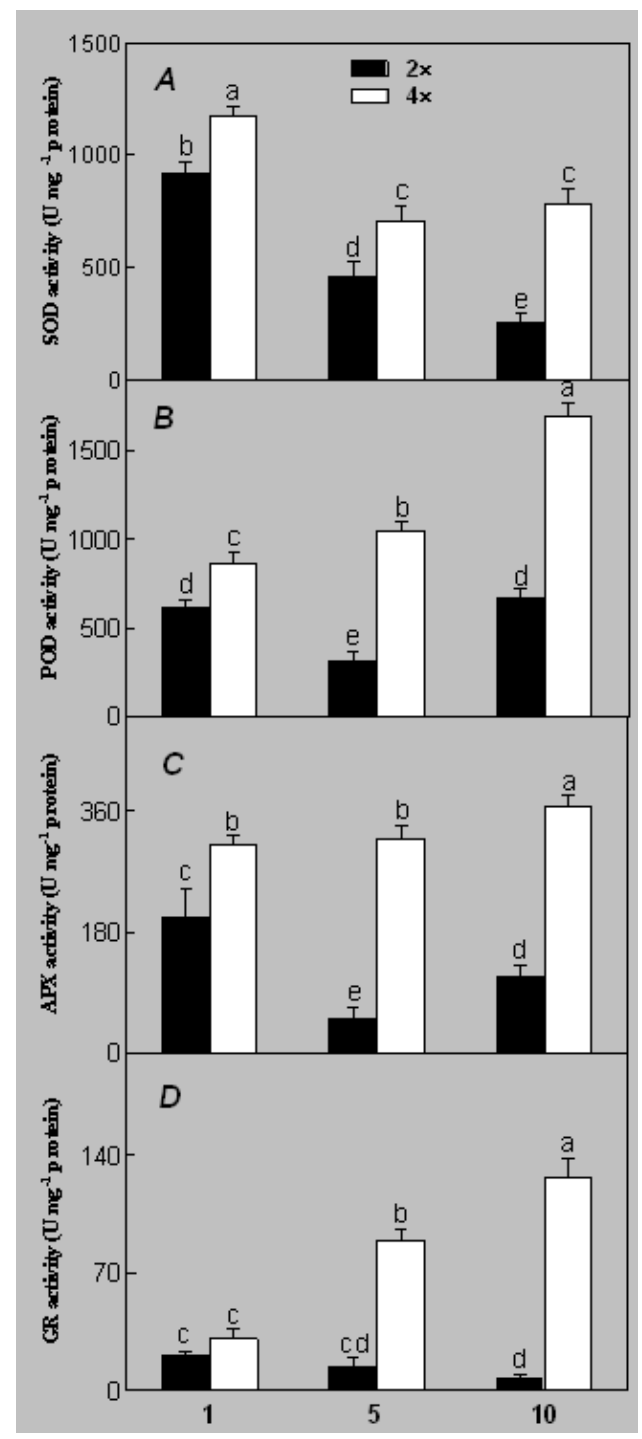

The results for the physiological responses of plant growth to salt stress were different (Figures 1-8). Salt stress inhibited plant growth and decreased net photosynthetic rate and stomatal conductance in $2 \times$. However, the decreases in these parameters were less in $4 \times$ than those in $2 \times$. These results agree with our earlier research showing that $4 \times$ plants are relatively salt-tolerant compared to that of 
$2 \times$ plants [24]. Similar results were reported by another group [25]. In Figure 5, our results showed that $P n$ and $G s$ for $4 \times$ were not sensitive in response to salt stress after RWC decreases are detectable. It may be a reason that the salt stress caused less pronounced inhibition of photosynthesis in $4 \times$ than in $2 \times$. In other words, $4 \times$ plants have higher water use efficiency under salt stress. Thus, $4 \times$ can keep photosynthesis stability under low RWC to adapt salt stress. At the same time, RWC is not only factor influencing $P n$. The decrease in growth and stomatal conductance may result from reduced leaf water content. Gs plays an important role in the change in $P n$. Little change in $C i$ was observed compared with that of Gs (Figure 3). This indicates that photosynthesis under salt stress may be affected by other factors such as the availability of ATP and Rubisco activity [26]. The changes between $P n, G s$, and $C i$ in $2 \times$ and $4 \times$ plants were different, which was further supported by the proteomics results. The expression levels of 19 and 16 proteins including Rubisco large subunit, rubisco activase and ATP synthase CF1 alpha subunit increased in $2 \times$ and $4 \times$ after salt treatment, respectively. MDA and REL are important indicators of the damage caused by salt stress [27]. The present results show that salinity significantly increased MDA content and REL in $2 \times$ more than those in $4 \times$, indicating more damage to membranes in $2 \times$ plants.

Salt stress induces the accumulation of reactive oxygen species (ROS) such as $\mathrm{H}_{2} \mathrm{O}_{2}$ and $\mathrm{O}_{2}{ }_{2}^{-}$. Excess accumulation of ROS causes oxidative damage to membrane lipids, nucleic acids, and proteins [28]. $\mathrm{H}_{2} \mathrm{O}_{2}$ and $\mathrm{O}_{2}{ }^{-}$accumulated in leaves of $2 \times$ and $4 \times$ plants after salt treatment. In general, plants reduce or scavenge ROS through antioxidant enzymes such as SOD, POD, APX, and GR. Interestingly, the activities of the antioxidant enzymes (POD, APX and GR) in $4 \times$ plants increased under salt stress. All antioxidant enzymes play an important role adjusting the cellular redox state [29]. In contrast, these enzymes (POD, APX and GR) decreased in $2 \times$. Antioxidant enzyme activities increase in most salt-tolerant plants [30]. However, SOD activity declined gradually in both $2 \times$ and $4 \times$ during salt treatment. Conversely, $4 \times$ plants showed higher SOD activity than that of $2 \times$, indicating that $4 \times$ has a more efficient enzymatic antioxidant system against salt stress and adjusting to ROS than those of $2 \times$ plants.

\subsection{Ultrastructural Responses of $2 \times$ and $4 \times$ Plants to Salt Stress}

The ultrastructure of the mesophyll cells of both $2 \times$ and $4 \times$ plants were similar after one day of salt treatment (Figure 7A,C). No significant differences were found in the chloroplasts $(C s)$ /thylakoid (Th) of the mesophyll cells of $2 \times$ and $4 \times$ plants after one day of salt treatment (Figure 8A,C). Nevertheless, the ultrastructure of $2 \times$ mesophyll cells changed noticeably after 10 days of salt treatment (Figure 7B). In $2 \times$, stress caused by severe salinity resulted in distorted $\mathrm{Cs}$, swollen $\mathrm{Th}$, and an accumulation in plastoglobules after 10 days of salt treatment. In addition, the number of starch grains increased (Figure 8B). However, the ultrastructural morphological injuries were not apparent in $4 \times$ salt-stressed plants (Figures 7D and 8D). 
Figure 7. Transmission electron micrographs of diploid $(2 \times)$ and tetraploid $(4 \times)$ Robinia pseudoacacia mesophyll cells after one day and 10 days of salt treatment. (A) 0 day, $2 \times$; (B) 0 day, 4×; (C) 10 days, 2×; (D) 10 days, 4×. CW, cell wall; Ch, chloroplast; M, mitochondrion; $\mathrm{Nu}$, nucleolus; $\mathrm{P}$, plastoglobule; $\mathrm{SG}$, starch granule; Gr, granum; V, vacuole; Vs, small vesicle.

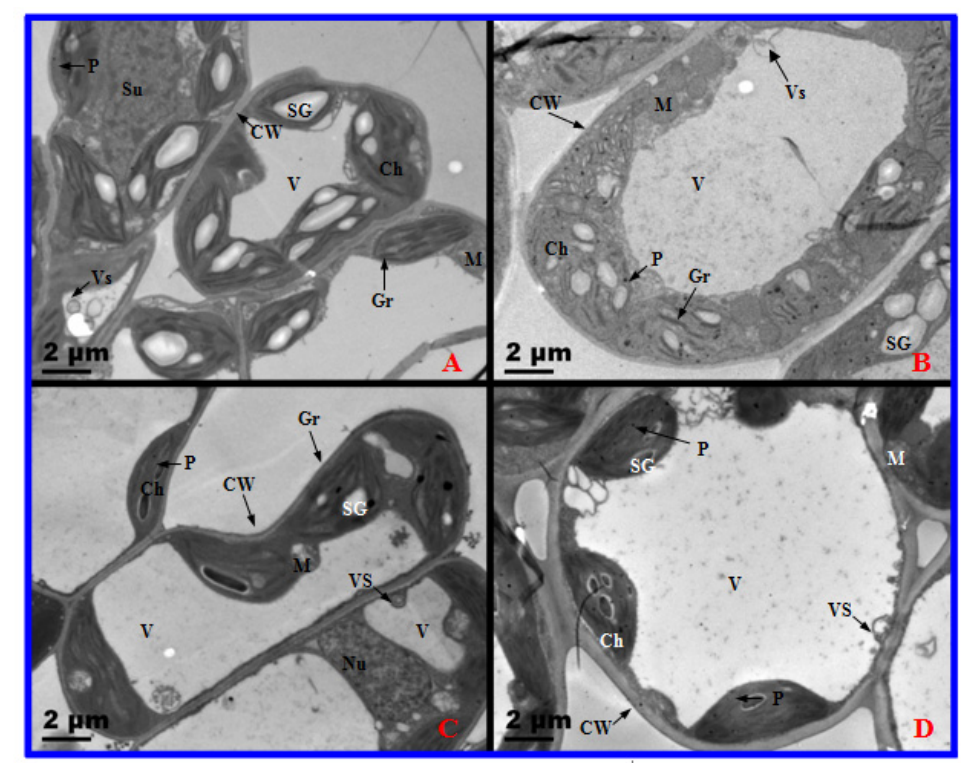

Figure 8. Transmission electron microscopy observations of chloroplasts in diploid $(2 \times)$ and tetraploid $(4 \times)$ Robinia pseudoacacia after one day and 10 days of salt treatment. (A) 0 day, 2×; (B) 10 days, 2×; (C) 0 day, 4×; (D) 10 days, 4×. Gr, granum; thylakoid (Th); $\mathrm{P}$, plastoglobule; $\mathrm{SG}$, starch grain.

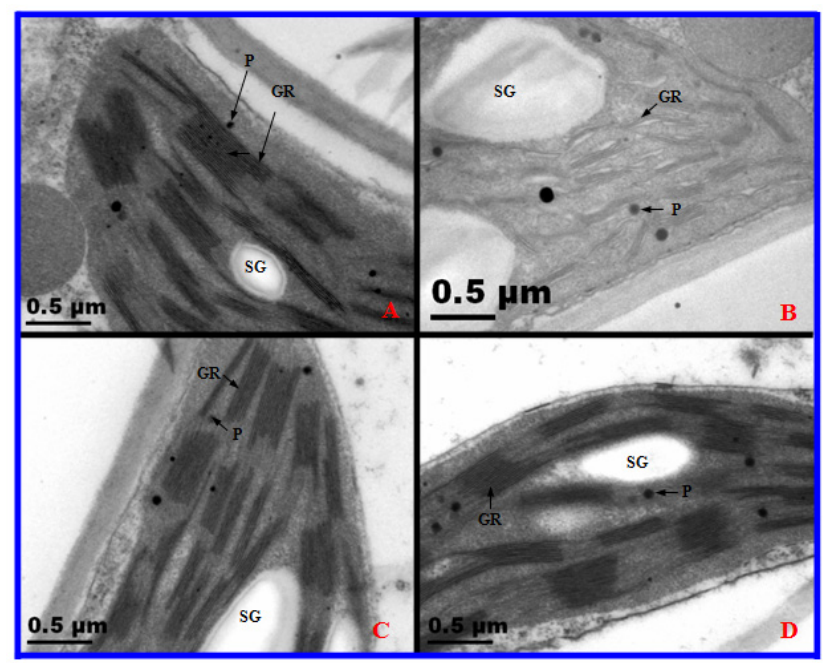

Salinity stress affects cell ultrastructure in many plant species. Photosynthesis occurs in chloroplasts; hence, it is the most severely impacted organelle under salt stress. Salinity induces severe disorganization in chloroplasts, leading to distorted chloroplasts and dilated thylakoid membranes in mesophyll cells of A. littoralis [31]. However, in this study, chloroplasts of $2 \times$ became swollen, plastoglobules and starch granules accumulated, and thylakoids exhibited disorganization after salt treatment (Figures 7 and 8). In addition, $2 \times$ plants showed a decline in $P n, G s, C i$, growth rate, and an 
increase in REL. These results reveal that salt stress destroyed the structure and function of mesophyll cells. Some reports have shown that disorganized chloroplasts are the main source of reactive oxygen and are involved in leaf senescence [32]. Therefore, $2 \times$ displayed accelerated senescence under salt stress. However, less serious injury was observed in chloroplasts of salt-stressed $4 \times$ than that in salt-stressed $2 \times$ at the same treatment stage, which again suggests that $2 \times$ suffered from greater negative effects than that of $4 \times$ when grown under high salinity conditions. Therefore, $4 \times$ can keep stable photosynthesis by organized cell ultrastructure.

\subsection{Identification of Differentially Expressed Proteins after Salt Treatment by Two-Dimensional} Electrophoresis (2-DE)

To investigate the changes in protein profiles under salt stress, total protein from the leaves of control and salt-treated plants was extracted and analyzed by 2-DE. Approximately 800 protein spots were detected in $2 \times$ and $4 \times$ plants, respectively. Master 2-D gel maps are shown in Figures 9 and 10. All protein spots were quantitatively analyzed, but only the protein spots that showed two-fold or more expression $(p<0.05)$ changes under the salt treatments were submitted for protein identification. All 41 protein spots from $2 \times$ gels and 37 protein spots from $4 \times$ gels were detected as differentially expressed spots (Figures 9 and 10; Tables 1 and 2). Twenty-eight proteins were up-regulated in $2 \times$ plants. Of which 13 proteins increased gradually in abundance in salt-treated plants, whereas 13 proteins increased in expression after 5 days of salt treatment and then decreased after 10 day of salt treatment. Two proteins decreased and then increased under salt stress compared to that in controls. Twenty-five proteins were up-regulated in $4 \times$ plants. Among them, 21 proteins increased gradually compared to controls and only four increased and then decreased with salt treatment.

Figure 9. Coomassie Brilliant Blue (CBB)-stained two-dimensional electrophoresis gels of proteins from Robinia pseudoacacia diploid $(2 \times)$ leaves. Proteins were separated on a 13 cm IPG strip ( $\mathrm{pH}$ 4-7 linear gradient) using isoelectric focusing, followed by sodium dodecyl sulfate polyacrylamide gel electrophoresis on a $12.5 \%$ gel. (A) 1-day NaCl-treated leaves; (B) 5-day NaCl-treated leaves; (C) 10-day NaCl-treated leaves. Blue and red numbers indicate proteins that increased and decreased between control and stressed samples, respectively. The proteins are listed in Table 1.

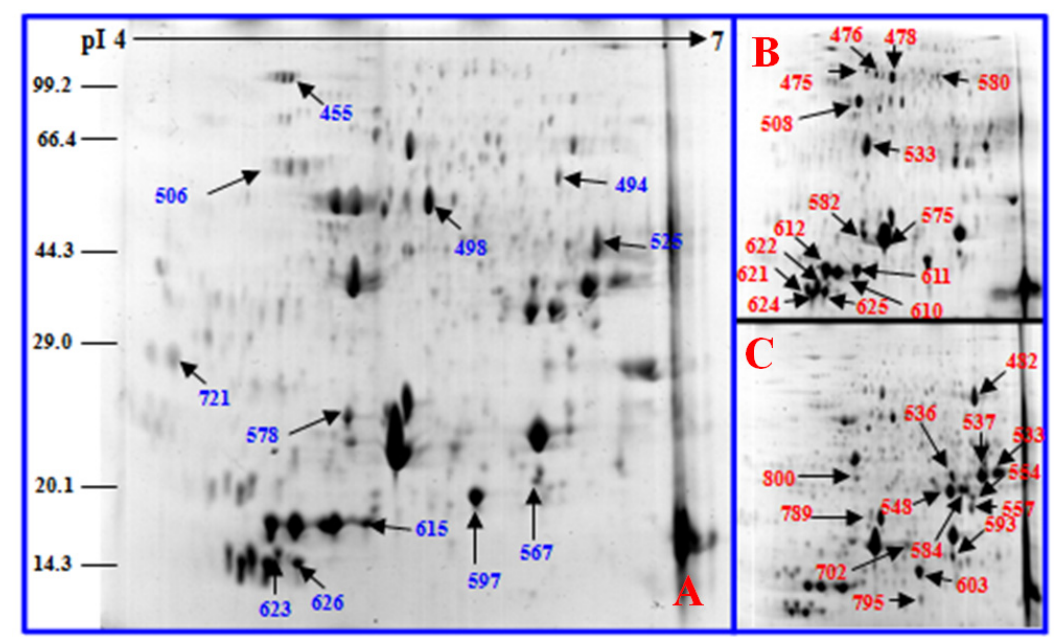


Figure 10. Coomassie Brilliant Blue (CBB)-stained two-dimensional electrophoresis gels of proteins from Robinia pseudoacacia tetraploid $(4 \times)$ leaves. Proteins were separated on $13 \mathrm{~cm}$ IPG strip (pH 4-7 linear gradient) using isoelectric focusing, followed by sodium dodecyl sulfate polyacrylamide gel electrophoresis on a $12.5 \%$ gel. (A) 1-day NaCl-treated leaves; (B) 5-day NaCl-treated leaves; (C) 10-day NaCl-treated leaves. Blue and red numbers indicate proteins that increased and decreased between control and stressed samples, respectively. The proteins are listed in Table 2.

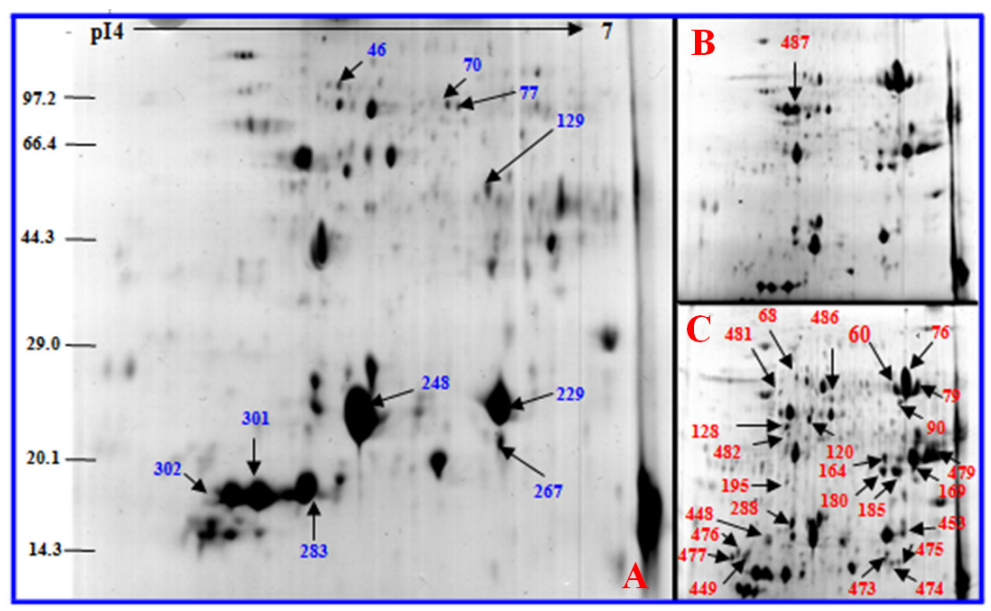

To further examine the differentially expressed proteins, all 78 protein spots were excised, digested, and submitted for protein identification. Based on the data from BLASTP, Gene Ontology, and some literature, the identified proteins covered a wide range of molecular functions (Tables 1 and 2). In $2 \times$, 41 identified proteins were classified into seven categories (Figure 11). The largest group of proteins was associated with photosynthesis (27), followed by stress and defense (5), metabolism (4), energy (2), transcription/translation related (1), transportation (1), and unclear classification (1). In 4×, 39 identified proteins were also classified into seven groups (Figure 12). Most of the proteins were involved in photosynthesis (23), followed by stress and defense (4), metabolism (3), energy (2), transportation (2), transcription/translation related (1), and unclear classification (2).

Figure 11. Functional categorization of proteins in Robinia pseudoacacia diploid $(2 \times)$ plants under salt stress. Digits indicate the protein number of each functional category.

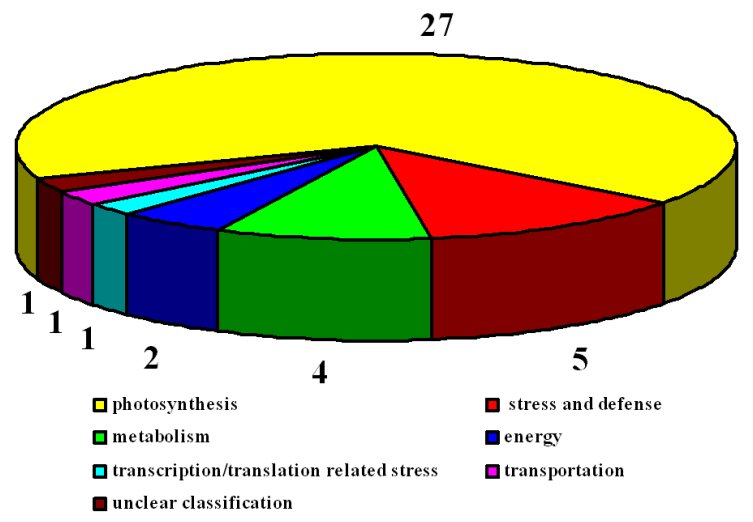


Table 1. Protein identities and their relative changes in leaves of tetraploid Robinia pseudoacacia after salt stress.

\begin{tabular}{|c|c|c|c|c|c|c|c|c|c|}
\hline $\begin{array}{l}\text { Spot } \\
\text { No. }^{a} \\
\end{array}$ & Protein Name & Species & gi Number ${ }^{b}$ & $\begin{array}{c}\text { Theoretical } \\
\text { MW(Da)/pI }{ }^{\mathbf{c}}\end{array}$ & $\begin{array}{l}\text { Experimental } \\
\mathrm{MW}(\mathrm{Da}) / \mathbf{p I}^{\mathrm{d}}\end{array}$ & Score $^{\mathrm{e}}$ & $\mathbf{M}^{\mathrm{f}}$ & $\mathrm{C}(\%)^{\mathrm{g}}$ & $\begin{array}{c}V \% \pm \mathrm{SE}^{\mathrm{h}} \\
(1 / 5 / 10 \text { days })\end{array}$ \\
\hline \multicolumn{10}{|c|}{ Energy } \\
\hline 494 & phosphoglycerate kinase & N. benthamiana & 313585890 & $50.05 / 7.66$ & $58.00 / 6.34$ & 172 & 10 & 19 & \\
\hline 537 & phosphoglycerate kinase & R. pseudoacacia & 2257598 & $23.67 / 6.49$ & $45.66 / 6.32$ & 479 & 34 & 100 & \\
\hline \multicolumn{10}{|c|}{ Metabolism } \\
\hline 498 & putative plastidic glutamine synthetase & O. sativa (japonica group) & 115461066 & $47.56 / 5.96$ & $51.16 / 5.52$ & 98 & 13 & 25 & \\
\hline 508 & chalcone synthase & R. pseudoacacia & 194740616 & $32.58 / 5.75$ & $50.19 / 5.09$ & 115 & 13 & 46 & \\
\hline 789 & aconitate hydratase domain protein & A. cellulolyticus $\mathrm{CD} 2$ & 303239527 & $19.84 / 4.86$ & $28.47 / 4.45$ & 91 & 11 & 58 & \\
\hline 536 & pyruvate kinase & C. reinhardtii & 159485206 & $23.201 / 6.2$ & $40.20 / 6.22$ & 86 & 23 & 15 & \\
\hline \multicolumn{10}{|c|}{ Photosynthesis } \\
\hline 506 & Rubisco large subunit & G. subaequalis & 24634972 & $52.37 / 6.34$ & $50.37 / 5.13$ & 215 & 31 & 45 & \\
\hline 538 & Rubisco large subunit & Caesalpinia sp. SH-2010 & 306481385 & $43.78 / 7.38$ & $45.71 / 6.67$ & 310 & 32 & 49 & \\
\hline 554 & Rubisco large subunit & Parkia multijuga & 148590322 & $51.78 / 6.23$ & $34.27 / 6.41$ & 190 & 19 & 27 & \\
\hline 557 & Rubisco large subunit & Merremia hastata & 21634009 & $47.96 / 6.42$ & $32.77 / 6.30$ & 216 & 18 & 25 & \\
\hline 575 & Rubisco large subunit & Dendrobium aphyllum & 300250366 & $22.77 / 6.2$ & $22.67 / 5.39$ & 217 & 13 & 54 & \\
\hline 582 & Rubisco large subunit & Loxocarya gigas & 5737828 & $49.48 / 6.43$ & $21.50 / 5.29$ & 258 & 11 & 26 & \\
\hline 584 & Rubisco large subunit & Ipomoea purpurea & 157325538 & $53.35 / 6.41$ & $37.47 / 6.26$ & 182 & 18 & 31 & \\
\hline 593 & Rubisco large subunit & Millettia lenneoides & 18032763 & $51.53 / 6.04$ & $24.01 / 6.22$ & 239 & 18 & 24 & \\
\hline
\end{tabular}


Table 1. Cont.

\begin{tabular}{|c|c|c|c|c|c|c|c|c|c|}
\hline $\begin{array}{l}\text { Spot } \\
\text { No. }{ }^{a} \\
\end{array}$ & Protein Name & Species & gi Number ${ }^{b}$ & $\begin{array}{c}\text { Theoretical } \\
\text { MW(Da)/pI }\end{array}$ & $\begin{array}{l}\text { Experimental } \\
\mathrm{MW}(\mathrm{Da}) / \mathrm{pI}^{\mathrm{d}}\end{array}$ & Score ${ }^{\mathrm{e}}$ & $\mathbf{M}^{\mathrm{f}}$ & $\mathrm{C}(\%)^{\mathrm{g}}$ & $\begin{array}{c}\mathrm{V} \% \pm \mathrm{SE}^{\mathrm{h}} \\
(1 / 5 / 10 \text { days })\end{array}$ \\
\hline 603 & Rubisco large subunit & R. pseudoacacia & 2343004 & $49.20 / 6.13$ & $19.78 / 5.88$ & 336 & 31 & 54 & \\
\hline 610 & Rubisco large subunit & Haematoxylum brasiletto & 66735773 & $18.40 / 6.05$ & $17.95 / 5.17$ & 179 & 14 & 46 & \\
\hline 612 & Rubisco large subunit & Marila laxiflora & 49823207 & $21.44 / 5.91$ & $18.190 / 4.98$ & 282 & 12 & 35 & \\
\hline 615 & Rubisco large subunit & R.pseudoacacia & 340511916 & $50.20 / 6.14$ & $18.69 / 5.28$ & 305 & 32 & 53 & \\
\hline 621 & Rubisco large subunit & Prunus salicina & 15987094 & $51.31 / 6.99$ & $16.61 / 4.30$ & 147 & 14 & 20 & \\
\hline 622 & Rubisco large subunit & Cecropia palmata & 6983898 & $52.00 / 6.23$ & $16.72 / 4.54$ & 126 & 13 & 16 & \\
\hline 623 & Rubisco large subunit & R. pseudoacacia & 2342974 & $52.01 / 6.14$ & $15.83 / 5.07$ & 116 & 15 & 28 & \\
\hline 624 & Rubisco large subunit & Stigmaphyllon paralias & 14599610 & $52.24 / 6.23$ & $15.23 / 4.41$ & 175 & 21 & 31 & \\
\hline 626 & Rubisco large subunit & Codonopsis dicentrifolia & 194400582 & $50.82 / 6.19$ & $15.03 / 4.96$ & 118 & 15 & 30 & \\
\hline 702 & Rubisco large subunit & Millettia lenneoides & 18032763 & $51.53 / 6.04$ & $22.37 / 5.72$ & 271 & 23 & 47 & \\
\hline 721 & Rubisco large subunit & Parthenocissus himalayana & 16973408 & $51.68 / 6.34$ & $31.78 / 4.26$ & 196 & 15 & 40 & \\
\hline 795 & Rubisco large subunit & R. pseudoacacia & 67079090 & $25.32 / 6.23$ & $16.84 / 5.84$ & 354 & 25 & 55 & \\
\hline 567 & Ribulose-bisphosphate carboxylase & Mangifera indica & 7261036 & $24.43 / 6.71$ & $27.20 / 6.56$ & 227 & 19 & 64 & \\
\hline 597 & Ribulose-bisphosphate carboxylase & Centrosema sp. SH-2010 & 306481395 & $49.81 / 6.44$ & $22794 / 6.23$ & 167 & 18 & 27 & \\
\hline 548 & Ribulose-biphosphate carboxylase oxygenase & Liparia genistoides & 146188483 & $27.28 / 6.36$ & $37.36 / 6.06$ & 263 & 25 & 60 & \\
\hline 533 & photosystem II protein $33 \mathrm{kD}$ & Spinacia oleracea & 224916 & $26.65 / 5.01$ & $38.28 / 5.34$ & 294 & 22 & 78 & \\
\hline
\end{tabular}


Table 1. Cont.

\begin{tabular}{|c|c|c|c|c|c|c|c|c|c|}
\hline $\begin{array}{l}\text { Spot } \\
\text { No. }{ }^{\text {a }}\end{array}$ & Protein Name & Species & gi Number ${ }^{b}$ & $\begin{array}{c}\text { Theoretical } \\
\text { MW(Da)/pI }\end{array}$ & $\begin{array}{l}\text { Experimental } \\
\mathrm{MW}(\mathrm{Da}) / \mathbf{p I}^{\mathrm{d}}\end{array}$ & Score $^{\mathrm{e}}$ & $\mathbf{M}^{\mathbf{f}}$ & $\mathrm{C}(\%)^{\mathrm{g}}$ & $\begin{array}{c}V \% \pm S E^{h} \\
(1 / 5 / 10 \text { days })\end{array}$ \\
\hline 578 & polypeptide of the oxygen evolving complex of photosystem II & Sonneratia apetala & 146454492 & $24.99 / 5.61$ & $24.59 / 5.03$ & 95 & 6 & 26 & \\
\hline 475 & ATP synthase CF1 alpha subunit & R. communis & 339516150 & $55.52 / 5.22$ & $70.28 / 5.03$ & 340 & 26 & 31 & \\
\hline 476 & putative ATP synthase beta subunit & O. sativa (japonica group) & 56784991 & $45.94 / 5.33$ & $69.32 / 5.49$ & 106 & 22 & 51 & \\
\hline \multicolumn{10}{|c|}{ Transportation } \\
\hline 480 & aspartyl/glutamyl-tRNA(asn/gln) amidotransferase subunit b & Stigmatella aurantiaca DW4/3-1 & 310819540 & $53.54 / 5.56$ & $59.82 / 6.02$ & 74 & 11 & 23 & \\
\hline \multicolumn{10}{|c|}{ Transcription/translation related } \\
\hline 625 & maturase-like protein & Coursetia weberbaueri & 23664381 & $60.78 / 9.42$ & $15.06 / 4.94$ & 193 & 18 & 39 & \\
\hline \multicolumn{10}{|c|}{ Stress and defense } \\
\hline 455 & heat shock protein 70 & Cucumis sativus & 1143427 & $75.37 / 5.15$ & $100.32 / 4.83$ & 332 & 21 & 25 & \\
\hline 478 & agglutinin I polypeptide B & R. pseudoacacia & 4033451 & $31.19 / 6.14$ & $64.35 / 5.50$ & 117 & 14 & 69 & \\
\hline 482 & agglutinin I polypeptide B & R. pseudoacacia & 4033451 & $31.19 / 6.14$ & $60.65 / 6.19$ & 488 & 19 & 69 & \\
\hline 525 & plastidic aldolase & N. paniculata & 4827253 & $43.07 / 6.38$ & $46.90 / 6.47$ & 202 & 25 & 48 & \\
\hline 800 & phenylalanine ammonia lyase & R. pseudoacacia & 194740604 & $78.41 / 6.31$ & $58.78 / 5.46$ & 274 & 31 & 55 & \\
\hline \multicolumn{10}{|c|}{ Unclear classification } \\
\hline 611 & predicted protein & Micromonas sp. RCC299 & 171910308 & $31.90 / 5.47$ & $18.68 / 5.22$ & 280 & 31 & 62 & \\
\hline
\end{tabular}

${ }^{a}$ Assigned spot number as indicated in Figure 9. ${ }^{\mathrm{b}}$ Accession numbers according to the NCBIInr database. ${ }^{\mathrm{c}, \mathrm{d}}$ Theoretical (c) and experimental (d) masses (kDa) and pIs of identified proteins.

${ }^{\mathrm{e}}$ Mascot protein score reported after searching against the NCBInr database. Experimental values were calculated by Image Master 2D Platinum software. Theoretical values were retrieved from the protein database. ${ }^{\mathrm{f}}$ The number of unique peptides identified for each protein. ${ }^{\mathrm{g}}$ Sequence coverage. ${ }^{\mathrm{h}}$ Mean of relative protein abundance and standard error. Three treatments including 1,5 , and 10 days after $500 \mathrm{mM} \mathrm{NaCl}$ treatment were performed. 
Table 2. Protein identities and their relative changes in leaves of tetraploid Robinia pseudoacacia under salt stress.

\begin{tabular}{|c|c|c|c|c|c|c|c|c|c|}
\hline Spot No. ${ }^{a}$ & Protein Name & Species & gi Number ${ }^{b}$ & $\begin{array}{c}\text { Theoretical } \\
\text { MW(Da)/pI }{ }^{\mathbf{c}}\end{array}$ & $\begin{array}{l}\text { Experimental } \\
\mathrm{MW}(\mathrm{Da}) / \mathrm{pI}^{\mathrm{d}}\end{array}$ & Score $^{e}$ & $\mathbf{M}^{\mathrm{f}}$ & $\mathrm{C}(\%)^{\mathrm{g}}$ & $\begin{array}{c}V \% \pm \mathrm{SE}^{\mathrm{h}} \\
(\mathbf{1} / \mathbf{5} / 10 \text { days })\end{array}$ \\
\hline \multicolumn{10}{|c|}{ Energy } \\
\hline 60 & phosphoglycerate kinase & N. benthamiana & 313585890 & $50.05 / 7.66$ & $98.76 / 6.24$ & 169 & 13 & 23 & \\
\hline 476 & mitochondrial F1-ATPase beta subunit & Dimocarpus longan & 269914683 & $59.87 / 6.18$ & $22.47 / 4.62$ & 274 & 23 & 38 & \\
\hline \multicolumn{10}{|c|}{ Metabolism } \\
\hline 229 & chalcone synthase & R. pseudoacacia & 194740620 & $36.76 / 6.22$ & $27.61 / 6.24$ & 608 & 29 & 32 & \\
\hline 486 & chalcone synthase & R. pseudoacacia & 194740616 & $32.58 / 5.75$ & $68.39 / 5.71$ & 175 & 15 & 48 & \\
\hline 70 & enolase & Glycine max & 42521309 & $47.69 / 5.31$ & $92.57 / 5.73$ & 130 & 12 & 20 & \\
\hline \multicolumn{10}{|c|}{ Photosynthesis } \\
\hline 79 & Rubisco large subunit & R. pseudoacacia & 340511916 & $50.71 / 6.14$ & $98.81 / 6.59$ & 299 & 34 & 62 & \\
\hline 90 & Rubisco large subunit & Daviesia rhizomata & 18032753 & $51.67 / 6.14$ & $67.60 / 6.28$ & 418 & 37 & 63 & \\
\hline 164 & Rubisco large subunit & Gironniera subaequalis & 24634972 & $52.37 / 6.34$ & $44.69 / 6.22$ & 340 & 35 & 47 & \\
\hline 180 & Rubisco large subunit & Wisteria sp. & 2343020 & $51.49 / 6.13$ & $39.61 / 6.19$ & 294 & 20 & 32 & \\
\hline 185 & Rubisco large subunit & Canavalia rosea & 18157259 & $52.11 / 6.14$ & $39.79 / 6.24$ & 209 & 17 & 30 & \\
\hline 267 & Rubisco large subunit & R. pseudoacacia & 2343004 & $49.23 / 6.13$ & $22.59 / 6.28$ & 156 & 20 & 47 & \\
\hline 301 & Rubisco large subunit & Mascagnia stannea & 14599586 & $51.57 / 6.14$ & $19.08 / 4.81$ & 347 & 29 & 56 & \\
\hline 302 & Rubisco large subunit & Mascagnia stannea & 14599586 & $51.57 / 6.14$ & $18.99 / 4.64$ & 308 & 31 & 54 & \\
\hline 448 & Rubisco large subunit & Floerkea proserpinacoides & 38147280 & $51.57 / 5.87$ & $24.53 / 4.89$ & 120 & 14 & 23 & \\
\hline
\end{tabular}


Table 2. Cont.

\begin{tabular}{|c|c|c|c|c|c|c|c|c|c|}
\hline Spot No. ${ }^{a}$ & Protein Name & Species & gi Number ${ }^{b}$ & $\begin{array}{c}\text { Theoretical } \\
\text { MW(Da)/pI }{ }^{\mathrm{c}}\end{array}$ & $\begin{array}{l}\text { Experimental } \\
\mathrm{MW}(\mathrm{Da}) / \mathbf{p I}^{\mathrm{d}}\end{array}$ & Score ${ }^{\mathrm{e}}$ & $\mathbf{M}^{\mathrm{f}}$ & $\mathrm{C}(\%)^{\mathrm{g}}$ & $\begin{array}{c}\mathrm{V} \% \pm \mathrm{SE}^{\mathrm{h}} \\
(\mathbf{1} / \mathbf{5} / \mathbf{1 0} \text { days }) \\
\end{array}$ \\
\hline 449 & Rubisco large subunit & Aspicarpa sericea & 331690047 & $49.79 / 6.13$ & $22.68 / 4.81$ & 175 & 16 & 27 & \\
\hline 453 & Rubisco large subunit & Wisteria sp. & 2343020 & $51.49 / 6.13$ & $27.71 / 6.32$ & 311 & 26 & 42 & \\
\hline 473 & Rubisco large subunit & Centrosema sp. SH-2010 & 306481395 & $49.81 / 6.44$ & $22.49 / 6.20$ & 265 & 27 & 31 & \\
\hline 474 & Rubisco large subunit & Sassafras albidum & 283558279 & $20.64 / 6.05$ & $21.07 / 6.24$ & 132 & 14 & 32 & \\
\hline 477 & Rubisco large subunit & Diospyros pentamera & 221078519 & $51.98 / 6.14$ & $21.06 / 4.35$ & 179 & 15 & 20 & \\
\hline 479 & Rubisco large subunit & Mucuna macrocarpa & 18157295 & $52.11 / 6.14$ & $44.05 / 6.82$ & 284 & 33 & 43 & \\
\hline 272 & Rubisco large subunit & Pachynema junceum & 9909908 & $19.07 / 5.33$ & $20.07 / 5.68$ & 226 & 14 & 36 & \\
\hline 283 & Rubisco large subunit & Pachynema junceum & 9909908 & $19.19 / 5.33$ & $19.14 / 5.18$ & 199 & 14 & 36 & \\
\hline 481 & Rubisco activase & Glycine max & 290766481 & $52.64 / 5.54$ & $98.57 / 5.11$ & 211 & 29 & 43 & \\
\hline 487 & Rubisco activase & Glycine max & 290766485 & $48.64 / 6.28$ & $53.18 / 5.47$ & 172 & 14 & 21 & \\
\hline 129 & Rubisco activase & Zantedeschia aethiopica & 13430334 & $37.25 / 6.7$ & $55.76 / 6.03$ & 157 & 18 & 35 & \\
\hline 68 & ATP synthase CF1 alpha subunit & R. communis & 339516150 & $55.52 / 5.22$ & $93.78 / 5.40$ & 472 & 28 & 36 & \\
\hline 475 & ATP synthase CF1 alpha subunit & Vigna radiata & 289066833 & $55.68 / 5.21$ & $21.06 / 6.24$ & 366 & 28 & 35 & \\
\hline 120 & Phosphoribulokinase (PPK) & Pisum sativum & 1885326 & $39.00 / 5.41$ & $58.67 / 5.57$ & 129 & 10 & 25 & \\
\hline
\end{tabular}


Table 2. Cont.

\begin{tabular}{|c|c|c|c|c|c|c|c|c|c|}
\hline Spot No. ${ }^{a}$ & Protein Name & Species & gi Number ${ }^{b}$ & $\begin{array}{c}\text { Theoretical } \\
\text { MW(Da)/pI }\end{array}$ & $\begin{array}{l}\text { Experimental } \\
\mathrm{MW}(\mathrm{Da}) / \mathbf{p I}^{\mathrm{d}}\end{array}$ & Score ${ }^{\mathrm{e}}$ & $\mathbf{M}^{\mathbf{f}}$ & $\mathrm{C}(\%)^{\mathrm{g}}$ & $\begin{array}{c}\mathrm{V} \% \pm \mathrm{SE}^{\mathrm{h}} \\
(\mathbf{1} / \mathbf{5} / \mathbf{1 0} \text { days })\end{array}$ \\
\hline \multicolumn{10}{|c|}{ Transportation } \\
\hline 46 & Rubisco subunit binding-protein beta subunit & R. communis & 255564820 & $64.15 / 5.65$ & $100.08 / 5.40$ & 127 & 13 & 23 & \\
\hline 248 & General secretion pathway protein D precursor, putative & R. communis & 255619353 & $30.81 / 4.67$ & $27.01 / 5.53$ & 174 & 17 & 54 & \\
\hline \multicolumn{10}{|c|}{ Transcription/translation related } \\
\hline 77 & maturase-like protein & Olneya tesota & 23477700 & $60.67 / 9.45$ & $91.49 / 6.01$ & 409 & 27 & 52 & \\
\hline \multicolumn{10}{|c|}{ Stress and defense } \\
\hline 76 & $\begin{array}{l}\text { Chain A, legume lectin sf the bark of robinia } \\
\text { pseudoacacia }\end{array}$ & R. pseudoacacia & 15826665 & $25.58 / 4.48$ & $99.77 / 6.42$ & 121 & 13 & 57 & \\
\hline 128 & sedoheptulose-1,7-bisphosphatase(SBPase) & Cucumis sativus & 229597543 & $42.08 / 5.96$ & $55.09 / 5.22$ & 99 & 11 & 23 & \\
\hline 169 & phenylalanine ammonia lyase & R. pseudoacacia & 194740606 & $34.40 / 5.4$ & $44.64 / 6.59$ & 300 & 21 & 72 & \\
\hline 195 & ascorbate peroxidase & Medicago sativa & 16304410 & $20.14 / 5.33$ & $32.49 / 5.26$ & 110 & 6 & 27 & \\
\hline \multicolumn{10}{|c|}{ Unclear classification } \\
\hline 482 & PREDICTED: ADP-ribosylation factor 1-like & Amphimedon queenslandica & 340369230 & $20.47 / 6.15$ & $49.38 / 5.26$ & 89 & 2 & 4 & \\
\hline 288 & Em protein & R. pseudoacacia & 1754977 & $12.22 / 6.21$ & $28.51 / 5.30$ & 356 & 22 & 98 & \\
\hline
\end{tabular}

${ }^{a}$ Assigned spot number as indicated in Figure $10 .{ }^{b}$ Accession numbers according to NCBIInr database. ${ }^{\mathrm{c}, \mathrm{d}}$ Theoretical (c) and experimental (d) mass (kDa) and pI of identified proteins. Mascot protein score reported after searching against the NCBInr database. Experimental values were calculated by Image Master 2D Platinum Software. Theoretical values were retrieved from the protein database. ${ }^{\mathrm{f}}$ The number of unique peptides identified for each protein. ${ }^{\mathrm{g}}$ Sequence coverage. ${ }^{\mathrm{h}}$ Mean of relative protein abundance and standard error. Three treatments including 1,5 and 10 days after $500 \mathrm{mM} \mathrm{NaCl}$ treatment were performed. 
Figure 12. Functional categorization of proteins in $R$. pseudoacacia tetraploid $(4 \times)$ plants under salt stress. Digits indicate the protein number of each functional category.

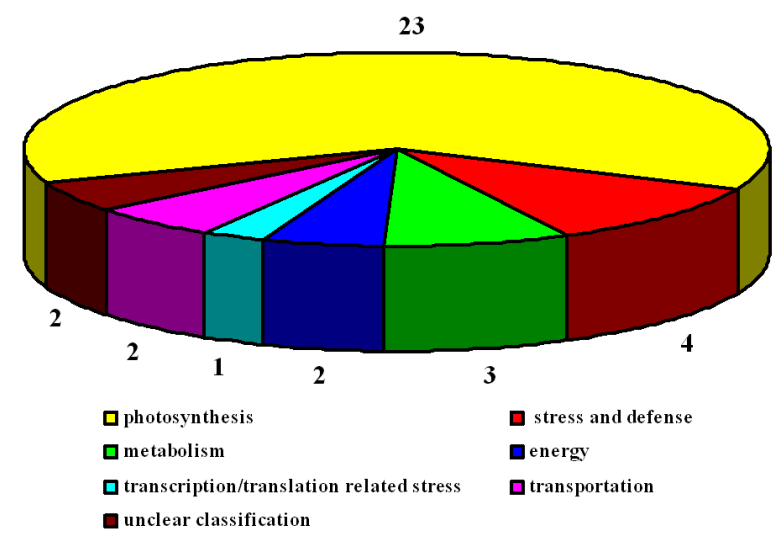

\subsubsection{Photosynthesis-Related Proteins}

Salt stress is a major environmental factor that limits the efficiency of photosynthesis. In our study, nearly half the proteins ( 27 in $2 \times$ and 23 in $4 \times$ ) were photosynthesis-related proteins. Among them, a number of proteins ( 20 in $2 \times$ and 17 in $4 \times$ ) were assigned to the Rubisco large subunit from different plant species but are visualized at positions with very different pIs and molecular weights (Tables 1 and 2). There results were not surprising because Rubisco is the most abundant protein in leaves. In addition, proteins in multiple spots can be translated from alternatively spliced mRNAs [33,34]. Two ribulose-bisphosphate carboxylase were only detectable in the treated $2 \times$, and three Rubisco activases were only detectable in the treated $4 \times$, suggesting that they responded specifically to salt stress. Among them, the two Rubisco activases increased in $4 \times$ after salt treatment. Previous reports also showed that the main role of this activase as an ATPase protein is maintenance of the catalytic activity of Rubisco by removing inhibitory sugars from the active site of uncarbamylated and carbamylated Rubisco [35,36]. Thus, increased Rubisico activase activity may have lead to increased photosynthetic rate $(P n)$ and increased growth rate. This result substantiated that $4 \times$ can tolerate high salt stress.

Photosystem II (PSII), as the core complex in photosynthesis, mediates oxygen evolution activity. PSII proteins (spot 578) in $2 \times$ were down-regulated under salt stress, suggesting that PSII activity was inhibited. These results were similar to previous observations [37]. In addition, two ribulose-bisphosphate carboxylase (RuBPCase) were down-regulated after salt treatment. RuBPCase is responsible for the primary step in $\mathrm{CO}_{2}$ fixation. Down-regulation of RuBPCase leads to a reduction in photosynthetic capacity. But, changes in these enzymes were not identified in $4 \times$ under salt stress. A possible reason is that $2 \times$ and $4 \times$ used different strategies to combat salt stress. Particularly, ATP synthase CF1 alpha subunit showed increased levels in $4 \times$ but decreased levels in $2 \times$ at 10 days of salt treatment. The increase in ATP synthase CF1 alpha subunit increases photosynthesis rate and down-regulates the Calvin cycle enzyme phosphoglycerate kinase [38]. Clearly, $4 \times$ had a greater capability to tackle salt stress by accumulating more photosynthesis-related proteins. 


\subsubsection{Stress and Defense Proteins}

Salt stress can lead to ion imbalance, hyperosmotic stress, and oxidative damage. Thus, plants induce various stress response proteins, many of which are crucial components of the plants self-defense network. Five and four proteins had altered translation levels in response to salinity stress in $2 \times$ and $4 \times$, respectively. Here, they were only visible in the treated $2 \times$ and $4 \times$ plants. Agglutinin I polypeptide $\mathrm{B}$ and phenylalanine ammonia lyase were significantly up-regulated after salt treatment in $2 \times$, whereas heat shock protein 70 and plastidic aldolase decreased under salt stress (Table 1). In $4 \times$, four proteins involved in the defense reaction, including legume lectin of the bark, sedoheptulose-1,7-bisphosphatase, phenylalanine ammonia lyase, and APX remarkably accumulated under salt stress (Table 2). The accumulation of proteins associated with defense could help $4 \times$ survive the high salt conditions. Our previous study showed that $4 \times$ plants have higher APX activity than that of $2 \times$ under salt stress. APX can detoxify $\mathrm{H}_{2} \mathrm{O}_{2}$ to $\mathrm{H}_{2} \mathrm{O}$ and plays an important role resisting salt stress in plants [39]. These results were not surprising because APX plays an important role in salt stress tolerance.

Lectins are carbohydrate-binding proteins expressed in plants upon exposure to biotic or abiotic stressors such as drought, salinity, heat, hormone treatment, pathogen attack, or insect herbivory. In the present study, the abundance of agglutinin I polypeptide B in $2 \times$ and chain A, a legume lectin of the bark in $4 \times$, increased after salt treatment. Lectins reduce the detrimental effects of salinity-induced oxidative stress in wheat seedlings, which is consistent with other findings [40]. Clearly, this suggests a very important role of lectins in plant salt tolerance.

\subsubsection{Energy Proteins}

When plants are exposed to high levels of salt, extra energy may be consumed to prevent damage. Phosphoglycerate kinase (PGK) and mitochondrial F1-ATPase beta subunits increased in $4 \times$ under salinity stress (Table 2). Some reports have revealed that PGK levels are enhanced by various stress conditions in several species [41]. PGK catalyzes the formation of ATP to ADP, which is essential for carbon fixation in plants. Enhancement of PGK would provide more ATP and ensure sufficient energy for plants to resist salt stress. In addition, the mitochondrial F1-ATPase beta subunit was up-regulated in $4 \times$ by salt treatment. Such up-regulation in the of F1-ATPase beta subunit protein level in response to salt treatment is likely to affect the tricarboxylic acid cycle, electron transport, and increase ATP synthesis. This may be one of the important mechanisms by which salt treatment reduces the extent of salinity-induced oxidative stress.

The same PGK (spot 494) was down-regulated in $2 \times$ under salt stress. However, another PGK was up-regulated. This was inconsistent with what was found in $4 \times$. This different response to salt stress between the two plants is interesting. However, the PGK protein was found in two spots, which is presumably due to post-transcriptional modification or proteolytic degradation of proteins in vivo and in vitro. 


\subsubsection{Metabolic Proteins}

Aconitate hydratase and pyruvate kinase are essential respiratory metabolism enzymes in plants. The abundance of aconitate hydratase and pyruvate kinase suggests that the tricarboxylic acid (TCA) and glycolysis increase under salinity stress. Enhancement of TCA and glycolysis would produce more ATP. Increases in aconitate hydratase and pyruvate kinase involved in respiratory metabolism showed the capacity of $2 \times$ plants to recover from salt stress. In contrast, no proteins associated with TCA or glycolysis changed significantly under salt stress in $4 \times$ plants. Besides, we found that the levels of chalcone synthase (CHS) and enolase changed remarkably in $4 \times$ under salt stress. A large number of investigations have shown that CHS and enolase are induced by various environmental stressors. Therefore, it was considered that $4 \times$ plants used another pathway to resist salt stress, or $4 \times$ as a halophyte had a high capacity to prevent damage resulting from oxidative stress.

In this study, to keep genetic stability, we propagated plants by cutting. Generally, cutting propagation can produce genetically identical progeny compared with hybrids propagation. Thus, cutting reproduction strongly influences genetic variation. Now, other methods such as flow cytometry and chromosome counting have been used for ploidy estimation (11).

\section{Experimental Section}

\subsection{Plant Materials and Growth Conditions}

All materials are introduced directly from South Korea to China by Beijing Forestry University. The diploid and tetraploid plants are from a germplasm. Thus, they have the same genetic origin. Thirty uniform plants (2-years-old) from $2 \times$ and $4 \times$ black locust were collected from Beijing Forestry University in Beijing, China and planted in plastic pots $(18 \mathrm{~cm}$ in diameter and $18 \mathrm{~cm}$ in depth) filled with 2:1 $(v / v)$ mixture of soil and sand. The experiments were carried out at Harbin Experimental Forest Farm of Northeast Forestry University in June 2011. Potted plants were grown in the greenhouse (day/night air temperature, $28 / 22{ }^{\circ} \mathrm{C}$, photoperiod $12 \mathrm{~h}$, and approximately $65 \%-85 \%$ relative humidity) for 1 month. Previous research showed that $4 \times$ tolerate high salt stress $(500 \mathrm{mM}$ $\mathrm{NaCl}$ ) for a short time. Thus, in this study, we treated plants with $500 \mathrm{mM} \mathrm{NaCl}$ for 15 days. After 1 , 5,10 , and 15 days of treatment, the leaves were harvested, immediately frozen in liquid nitrogen, and stored at $-80{ }^{\circ} \mathrm{C}$ prior to physiological and proteomic experiments. At least three independent biological experiments for each treatment were replicated.

\subsection{Morphological and Biomass Measurements}

The morphological traits of all plants were observed and photographed at 1, 5, 10, and 15 days of treatment. At the beginning and end of the salt treatments, height, range, and stem basal diameter of plants and leaf water content were recorded. The HGR, RGR, and the relative growth rate of stem basal diameter (BGR) were calculated using the following formulas: HGR $=\left(h_{2}^{\prime}-h_{1}^{\prime}\right) /\left(h_{2}-h_{1}\right)$, where $h_{1}^{\prime}$ is the height of salt-stressed plant at the beginning, $h_{2}^{\prime}$ is the height of salt-stressed plants at the end, $h_{1}$ is the height of control plants at the beginning, $h_{2}$ is the height of control plant at the end. $\mathrm{RGR}=\left(r_{2}^{\prime}-r_{1}^{\prime}\right) /\left(r_{2}-r_{1}\right)$, where $r_{1}^{\prime}$ is the range of stressed plants at the beginning, $r_{2}^{\prime}$ is the range of 
stressed plants at the end, $r_{1}$ is the range of control plants at the beginning, $r_{2}$ is the range of control plants at the end. BGR $=\left(b_{2}^{\prime}-b_{1}^{\prime}\right) /\left(b_{2}-b_{1}\right)$, where $b_{1}^{\prime}$ is the stem basal diameter of stressed plants at the beginning, $b^{\prime}{ }_{2}$ is the stem basal diameter of stressed plants at the end, $b_{1}$ is the stem basal diameter of control plants at the beginning, $b_{2}$ is the stem basal diameter of control plants at the end. At 9:30 AM, the fully expanded leaves were collected to measure relative water content (RWC) of leaves using the following formula: RWC $(\%)=(\mathrm{FW}-\mathrm{DW}) /(\mathrm{TW}-\mathrm{DW}) \times 100$, where $\mathrm{FW}$ is fresh weight, TW is turgid weight after rehydrating the samples for $24 \mathrm{~h}$, and DW is dry weight after oven-drying the samples at $85^{\circ} \mathrm{C}$ for $24 \mathrm{~h}$.

\subsection{Ion Content Analysis}

Leaves were dried at $80{ }^{\circ} \mathrm{C}$ and digested in nitric acid $\left(1 \% \mathrm{HNO}_{3}(v / v)\right)$ using the method of Wolf (1982) to measure $\mathrm{Na}^{+}$and $\mathrm{K}^{+}$[42]. $\mathrm{Na}^{+}$and $\mathrm{K}^{+}$were analyzed by flame emission using atomic absorption spectrophotometry (PerkinElmer Analyst 800, Waltham, MA, USA). $\mathrm{Cl}^{-}$was quantified by a modified silver titration method described by Chen et al. (2001) [43].

\subsection{Determination of Superoxide Radical, Hydrogen Peroxide, Lipid Peroxidation and Relative Electrolyte Leakage}

Leaves $(0.5 \mathrm{~g})$ were ground at $4{ }^{\circ} \mathrm{C}$ and then homogenized in $5 \mathrm{~mL} 50 \mathrm{mM}$ potassium phosphate buffer ( $\mathrm{pH} 7.8)$ containing $0.1 \mathrm{mM}$ EDTA, $1 \%(w / v)$ PVP, $0.1 \mathrm{mM}$ PMSF, and $0.2 \%(v / v)$ Triton $\mathrm{X}-100$. The mixture was centrifuged at $12,000 \times \mathrm{g}$ for $15 \mathrm{~min}$ at $4{ }^{\circ} \mathrm{C}$. One millilitre the supernatant was mixed with a mixture of $1 \mathrm{~mL}$ hydroxylamine hydrochloride for $1 \mathrm{~h}, 1 \mathrm{~mL} \beta$-aminobenzene sulfonic acid, and $1 \mathrm{~mL} \alpha$-naphthylamine, and then the solution was incubated at $25{ }^{\circ} \mathrm{C}$ for $20 \mathrm{~min}$. The concentration of superoxide radical was determined by measuring the absorbance of the mixture at $530 \mathrm{~nm}$ using a $\mathrm{NaNO}_{2}$ standard curve. Hydrogen peroxide $\left(\mathrm{H}_{2} \mathrm{O}_{2}\right)$ content was determined by measuring the absorbance of the $\mathrm{H}_{2} \mathrm{O}_{2}$ titanium complex at $410 \mathrm{~nm}$ using known concentrations of $\mathrm{H}_{2} \mathrm{O}_{2}$ as the standard curve. Lipid peroxidation was estimated as MDA content using a modified method. Leaves $(0.2 \mathrm{~g})$ were homogenized in $5 \mathrm{~mL}$ of $5 \%$ of trichloroacetic acid (TCA) and centrifuged at $12,000 \times \mathrm{g}$ for $15 \mathrm{~min}$. Two $\mathrm{ml}$ of supernatant was added to a test tube containing the mixture of $2 \mathrm{~mL}$ of $20 \%$ TCA, $0.01 \%$ butylated hydroxytoluene, and $0.6 \%$ thiobarbituric acid. The mixture was heated in boiling water for $30 \mathrm{~min}$, and then quickly cooled on ice. After centrifugation at $12,000 \times \mathrm{g}$ for $10 \mathrm{~min}$, the absorbance of the supernatant was determined at 450, 532 and $600 \mathrm{~nm}$.

Twenty leaf discs $\left(1.0 \mathrm{~cm}^{2}\right)$ from the third to fifth fully expanded leaves were vacuum-infiltrated in $10 \mathrm{~mL}$ deionized water for $30 \mathrm{~min}$ and maintained in water for $6 \mathrm{~h}$. Using a portable conductivity detector (LC116, Mettler Toledo Instruments Co., Ltd., Shanghai, China), the conductivity of the bathing solution (C1) was determined. Then, the leaf discs and the bathing solution were boiled for $10 \mathrm{~min}$ and thoroughly cooled to room temperature, and the conductivity of the resulting solution $(\mathrm{C} 2)$ was determined. The REL was calculated $(\operatorname{REL}(\%)=(\mathrm{C} 1 / \mathrm{C} 2) \times 100)$. 


\subsection{Photosynthesis Analysis}

Net photosynthetic rate $(P n), G s$, and intercellular $\mathrm{CO}_{2}$ concentration $(C i)$ were measured at 0,5 , and 10 days of salt treatment from 9:00 to 11:30 in the morning with a portable photosynthesis measuring system LI-COR 6400 (LI-COR Inc., Lincoln, NE, USA).

\subsection{Antioxidant Enzyme Activity Analysis}

SOD (EC1.15.1.1) activity was measured following the method of Roth and Gilbert (1984) [44]. The reaction mixture contained $20 \mu \mathrm{L}$ enzyme extract, $50 \mathrm{mM}$ sodium phosphate buffer ( $\mathrm{pH} 7.8$ ), $100 \mu \mathrm{M}$ EDTA, and $10 \mathrm{mM}$ pyrogallol. Enzyme activity was detected at $420 \mathrm{~nm}$ by spectrophotometer. POD (EC1.11.1.7) activity was measured according to the method of Nickel and Cunningham (1969) [45]. The reaction mixture contained $25 \mathrm{mM}$ PBS (pH 7.0), 0.05\% guaiacol, $10 \mathrm{mM} \mathrm{H}_{2} \mathrm{O}_{2}$, and enzyme extract. POD activity was measured at $470 \mathrm{~nm}$. GR (EC1.6.4.2) activity was assayed using the method of Nordhoff et al. (1993) [46]. GR activity was determined by NADPH oxidation at $340 \mathrm{~nm}$. The reaction mixture contained $10 \mu \mathrm{L}$ enzyme extract, $100 \mathrm{mM}$ potassium phosphate buffer ( $\mathrm{pH} 7.8$ ), $0.2 \mathrm{mM}$ NADPH, $2 \mathrm{mM}$ EDTA, and $0.5 \mathrm{mM}$ glutathione. The reaction was initiated by adding NADPH at $25^{\circ} \mathrm{C}$. The APX (EC1.11.1.11) activity assay was carried out using the method of Nakano and Asada (1981) [47]. The reaction mixture contained $50 \mathrm{mM}$ sodium phosphate buffer ( $\mathrm{pH} 7$ ) including $0.2 \mathrm{mM}$ EDTA, $0.5 \mathrm{mM}$ ascorbic acid, $50 \mathrm{mg}$ of BSA, and crude enzyme extract. The reaction was started by adding $\mathrm{H}_{2} \mathrm{O}_{2}$ at a final concentration of $0.1 \mathrm{mM}$.

\subsection{Ultrastructural Leaf Analysis}

Fresh leaf segments (about $1.5 \mathrm{~cm}$ in length and $0.5 \mathrm{~cm}$ in width) were fixed in $2.5 \%$ glutaral pentanedial $(v / v)$ at $4{ }^{\circ} \mathrm{C}$ for $2 \mathrm{~h}$, washed twice in 0.1 M PBS (sodium phosphate buffer, $\mathrm{pH}$ 6.8) at $4{ }^{\circ} \mathrm{C}$. Then, they were postfixed in $2 \%$ osmium tetraoxide $\left(\mathrm{O}_{\mathrm{s}} \mathrm{O}_{4}\right)$ for $2 \mathrm{~h}$, sequentially dehydrated in 50\%, 70\%, 90\%, and 100\% acetone, and embedded in Epon 812 for $2 \mathrm{~h}$. Ultra-thin sections (70 nm) were sliced, stained with uranyl acetate and lead citrate, and then mounted on copper grids for viewing on the H-600 IV TEM (Hitachi, Tokyo, Japan) at an accelerating voltage of $60 \mathrm{kV}$.

\subsection{Extraction of Total Leaf Protein}

Total leaf protein of plants at different treatment times $(0,5$, and 10 days) was extracted using the method of Hurkman and Tanaka (1986) [48]. Fresh leaf samples were ground in liquid nitrogen using a mortar and pestle to make a fine powder, and then suspended in 10\% ice-cold TCA in acetone containing $0.07 \% \beta$-mercaptoethanol. The suspension was allowed to precipitate overnight at $-20{ }^{\circ} \mathrm{C}$ and was centrifuged at $15,000 \times \mathrm{g}$ for $50 \mathrm{~min}$ at $4{ }^{\circ} \mathrm{C}$. The supernatant was removed, the precipitate was washed three times with ice-cold acetone containing $0.07 \% \beta$-mercaptoethanol for $1 \mathrm{~h}$ at $-20^{\circ} \mathrm{C}$, and the protein pellet was air-dried and stored at $-80{ }^{\circ} \mathrm{C}$. The protein powder was solubilized in lysis buffer (7 M urea, $2 \mathrm{M}$ thiourea, 4\% $w / v$ CHAPS, $40 \mathrm{mM}$ DTT, 2\% $\% / v \mathrm{pH} 4-7$ IPG buffer, and 4\% $w / v$ PMSF), and the supernatant was collected by centrifugation at $15,000 \times g$ for $50 \mathrm{~min}$ at $4{ }^{\circ} \mathrm{C}$. The protein content was assayed using bovine serum albumin as the standard according to the method of Bradford (1976) [49]. 


\subsection{2-DE Image Analysis and Gel Staining}

2-DE was carried out according to the method of Bjellqvist et al. (1982). A mixture of $300 \mu \mathrm{g}$ protein sample in $350 \mu \mathrm{L}$ of rehydration buffer containing $7 \mathrm{M}$ urea, $2 \mathrm{M}$ thiourea, $2 \% w / v$ CHAPS, $40 \mathrm{mM}$ DTT, and 0.5\% v/v IPG buffers, $\mathrm{pH} 4-7$ (GE Healthcare Bio-Sciences Corp., Piscataway, NJ, USA), and $0.01 \%$ bromophenol blue was prepared. The mixture was loaded onto IPG strips $(13 \mathrm{~cm}$, linear pH 4-7, GE Healthcare Bio-Sciences, Uppsala, Sweden). After overnight rehydration of the IPG strips at $20{ }^{\circ} \mathrm{C}$, isoelectric focusing was carried out on an Ettan IPGphorII (GE Healthcare, Bio-Sciences, Uppsala, Sweden) at $20{ }^{\circ} \mathrm{C}$. Isoelectric focusing was performed using the following procedure: $30 \mathrm{~V}$ for $2 \mathrm{~h}, 100 \mathrm{~V}$ for $1 \mathrm{~h}, 500 \mathrm{~V}$ for $1 \mathrm{~h}, 1000 \mathrm{~V}$ for $1 \mathrm{~h}$, gradient $8000 \mathrm{~V}$ for $0.5 \mathrm{~h}$, and $8,000 \mathrm{~V}$ rapid focus for $6 \mathrm{~h}$. After focusing, the strips were equilibrated by reduction with DTT and carboxymethylation with iodoacetamide. The second dimension was performed on $12.5 \%(w / v)$ polyacrylamide gels at $15 \mathrm{~mA}$ per gel for $30 \mathrm{~min}$ followed by $30 \mathrm{~mA}$ until the bromophenol blue ran off the bottom of the gel. Gels were stained with Coomassie Brilliant Blue R-250 and destained the next day.

Gel images were scanned using an ImageScanner III (GE Healthcare, Bio-Sciences, Uppsala, Sweden). Images were analyzed with ImageMaster 2D Platinum 7.0 software (Amersham Biosciences, Piscataway, NJ, USA, 2011). The average volume percent values were calculated from three technical replicates to represent the final volume percent values of each biological replicate. The experimental molecular mass and PI of the protein spots were determined by 2-DE standards and interpolation of missing values on the IPG strips. Spots were quantified based on total density of the gels by the percentage volume. Significantly different spots, which were determined as $p<0.05$ and a change of more than two-fold in abundance, were considered to be differentially accumulated proteins, and they had to be consistently present in three replications.

\subsection{In-Gel Digestion and Matrix-Assisted Time of Flight Mass Spectroscopy} (MALDI-TOF-MS) Analysis

Selected protein spots were excised, washed with $50 \%(v / v)$ acetonitrile in $0.1 \mathrm{M} \mathrm{NH}_{4} \mathrm{HCO}_{3}$, and dried at room temperature. Proteins were reduced with $1 \mathrm{mM}$ DTT and $2 \mathrm{mM} \mathrm{NH}_{4} \mathrm{HCO}_{3}$ at $55{ }^{\circ} \mathrm{C}$ for $1 \mathrm{~h}$ and alkylated with $55 \mathrm{mM}$ iodoacetamide in $25 \mathrm{mM} \mathrm{NH}_{4} \mathrm{HCO}_{3}$ in the dark at room temperature for $45 \mathrm{~min}$. The gel pieces were thoroughly washed with $25 \mathrm{mM} \mathrm{NH}_{4} \mathrm{HCO}_{3}, 50 \% \mathrm{ACN}, 100 \% \mathrm{ACN}$, and dried. The proteins were digested in $10 \mathrm{~mL}$ modified trypsin (Promega, Madison, WI, USA) solution ( $1 \mathrm{ng} \mathrm{mL} \mathrm{m}^{-1}$ in $25 \mathrm{mM}$ ammonium bicarbonate) during an overnight incubation at $37{ }^{\circ} \mathrm{C}$. Digests were immediately spotted onto $600 \mathrm{~mm}$ Anchorchips (Bruker Daltonics, Bremen, Germany). Spotting was achieved by pipetting $1 \mathrm{~mL}$ of analyte onto the MALDI target plate in duplicate and then adding $0.05 \mathrm{~mL}$ of $20 \mathrm{mg} \mathrm{mL}^{-1} \alpha-\mathrm{CHCA}$ in $0.1 \% \mathrm{TFA} / 33 \%(v / v) \mathrm{ACN}$, which contained $2 \mathrm{mM}$ ammonium phosphate. All samples were analyzed in the positive-ion reflectron mode on a TOF Ultraflex II mass spectrometer (Bruker Daltonics, Billerica, United states). Each acquired mass spectra ( $\mathrm{m} / \mathrm{z}$ range 700-4000, resolution 15,000-20,000) was processed using FlexAnalysis v2.4 software (Bruker Daltonics, Bremen, Gemeny, 2004). Proteins were identified with Mascot software (http://www.matrixscience.com) based on the mass signals to search for proteins in the SwissProt, NCBInr, and MSDB databases. 


\subsection{Statistical Analyses}

Statistical analyses were performed with SPSS 17.0 software (SPSS Inc., Chicago, IL, USA, 2009). All parameters are presented as mean \pm standard error and were obtained from at least three replicates and analyzed using Duncan's multiple range test or Student's $t$-test. A $p$-value $<0.05$ was considered significant.

\section{Conclusions}

Tetraploid black locust has high ecological and economic value. Until now, little was known about the implications of ploidy level in black locust on the physiological and proteomic responses under salt stress. In this study, the physiological and proteomic responses of $2 \times$ and $4 \times$ black locust were detected under salt stress during different times. Our results demonstrated that $2 \times$ plants suffered from greater negative effects than those of $4 \times$ plants according to their morphological and physiological characteristics under salt stress. In addition, 2-DE was used to analyze ploidy differences of the black locust leaf proteome under salt stress. Therefore, $4 \times$ plants have higher levels of several ROS scavenging enzymes and accumulate photosynthesis-related enzymes, defense protein and energy proteins to cope with salt stress compared with $2 \times$. These results contribute to selection of some specific proteins during ploidy process. In addition, the results suggested that $4 \times$ had a greater capability to defend against salt stress by accumulating more related proteins. Our results provide more information to further understand the molecular and physiological basis of stress tolerance in polyploid plants.

\section{Acknowledgments}

This study was supported the Fund for Fostering Talents in Basic Science of the National Natural Science Foundation of China (No. J1210053), the National Natural Science Foundation of China (31170568).

\section{Conflicts of Interest}

The authors declare no conflict of interest.

\section{References}

1. Horie, T.; Schroeder, J.I. Sodium transporters in plants. Diverse genes and physiological functions. Plant Physiol. 2004, 136, 2457-2462.

2. Zhu, J.K. Plant salt tolerance. Trends Plant Sci. 2001, 6, 66-71.

3. Srivastava, A.K.; Ramaswamy, N.K.; Mukopadhyaya, R.; Chiramal, J.M.G.; D’Souza, S.F. Thiourea modulates the expression and activity profile of mtATPase under salinity stress in seeds of Brassica juncea. Ann. Bot. 2009, 103, 403-410.

4. Yang, A.; Dai, X.; Zhang, W.H. A R2R3-type MYB gene, OsMYB2, is involved in salt, cold, and dehydration tolerance in rice. J. Exp. Bot. 2012, 63, 2541-2556.

5. Breyne, P.; Zabeau, M. Genome-wide expression analysis of plant cell cycle modulated genes. Curr. Opin. Plant Biol. 2001, 4, 136-142. 
6. Salekdeh, G.H.; Siopongco, J.; Wade, L.J.; Ghareyazie, B.; Bennett, J. Proteomic analysis of rice leaves during drought stress and recovery. Proteomics 2002, 2, 1131-1145.

7. Zhang, H.; Han, B.; Wang, T.; Chen, S.; Li, H.; Zhang, Y.; Dai, S. Mechanisms of plant salt response: Insights from proteomics. J. Proteome Res. 2012, 11, 49-67.

8. Romero-Aranda, R.; Bondada, B.R.; Syvertsen, J.P.; Grosser, J.W. Leaf characteristics and net gas exchange of diploid and autotetraploid citrus. Ann. Bot. 1997, 79, 153-160.

9. Wang, Q.L.; Yu, M.D.; Lu, C.; Wu, C.R.; Jing, C.R. Study on breeding and photosynthetic characteristics of new polyploidy variety for leaf and fruit-producing mulberry (Morus L). Sci. Agric. Sin. 2011, 44, 562-569.

10. Masterson, J. Stomatal size in fossil plants: Evidence for polyploidy in majority of angiosperms. Science 1994, 264, 421-424.

11. Stupar, R.M.; Bhaskar, P.; Yandell, B.; Rensink, W.A.; Hart, A.L.; Ouyang, S.; Veilleux, R.E.; Busse, J.S.; Erhardt, R.J.; Buell, C.R.; et al. Phenotypic and transcriptomic changes associated with potato autopolyploidization. Genetics 2007, 176, 2055-2067.

12. Riddle, N.C.; Jiang, H.; An, L.; Doerge, R.W.; Birchler, J.A. Gene expression analysis at the intersection of ploidy and hybridity in maize. Theor. Appl. Genet. 2010, 120, 341-353.

13. Ramsey, J. Polyploidy and ecological adaptation in wild yarrow. Proc. Natl. Acad. Sci. USA 2011, 108, 6697-6669.

14. Li, F.Z.; Ning, X.M.; Qiu, X.M.; Su, C.F.; Yao, J.Q.; Tian, L.W. Genetic mapping of the dark brown fiber $L c 1$ gene in tetraploid cotton. Sci. Agric. Sin. 2012, 45, 4109-4114.

15. Allario, T.; Brumos, J.; Colmenero-Flores, J.M.; Tadeo, F.; Froelicher, Y.; Talon, M.; Navarro, L.; Ollitrault, P.; Morillon, R. Large changes in anatomy and physiology between diploid Rangpur lime (Citrus limonia) and its autotetraploid are not associated with large changes in leaf gene expression. J. Exp. Bot. 2013, 62, 2507-2519.

16. Podda, A.; Checcucci, G.; Mouhaya, W.; Centeno, D.; Rofidal, V.; del Carratore, R.; Luro, F.; Morillon, R.; Ollitrault, P.; Maserti, B.E. Salt-stress induced changes in the leaf proteome of diploid and tetraploid mandarins with contrasting $\mathrm{Na}^{+}$and $\mathrm{Cl}^{-}$accumulation behaviour. J. Plant Physiol. 2013, 170, 1101-1112.

17. Beest, M.; le Roux, J.J.; Richardson, D.M.; Brysting, A.K; Suda, J.; Kubešová, M.; Pyšek, P. The more the better? The role of polyploidy in facilitating plant invasions. Ann. Bot. 2012, 109, 19-45.

18. Lu, C.; Cui, B.; Huang, L.; Sun, P.; Zhang, G.; Li, Y. Phenotypic observation and analysis of inflorescence variation of Autotetraploid Robinia pseudoacacia. Sci. Silvae Sin. 2012, 48, 63-68.

19. Al, H.A.; Monneveaux, P.; Nachit, M.M. Direct and indirect selection for drought tolerance in alien tetraploid wheat durum wheat crosses. Euphytica 1998, 100, 287-294.

20. Fock, I.; Collonnier, C.; Purwito, A.; Luisetti, J.; Souvannavong, V.; Vedel, F.; Servaes, A.; Ambroise, A.; Kodja, H.; Ducreux, G.; et al. Resistance to bacterial wilt in somatic hybrids between Solanum tuberosum and Solanum phureja. Plant Sci. 2000, 160, 165-176.

21. Huang, S.; Sirikhachornkit, A.; Su, X.; Faris, J.; Gill, B.S.; Haselkorn, R.; Gornicki, P. Genes encoding plastid acetyl-CoA carboxylase and 3-phosphoglycerate kinase of the Triticum/Aegilops complex and the evolutionary history of polyploid wheat. Proc. Natl. Acad. Sci. USA. 2002, 99, 8133-8138. 
22. Zhang, X.Y.; Hu, C.G.; Yao, J.L. Tetraploidization of diploid Dioscorea results in activation of the antioxidant defense system and increased heat tolerance. J. Plant Physiol. 2010, 167, 88-94.

23. Xiong, Y.C.; Li, F.M.; Zhang, T. Performance of wheat crops with different chromosome ploidy: Root-sourced signals, drought tolerance, and yield performance. Planta 2006, 224, 710-718.

24. Meng, F.J.; Huang, F.L. Changes of function and ultrastructure of mitochondria in Robinia pseudoacacia leaves under salt stress. Nonwood For. Res. 2010, 28, 18-23.

25. Saleh, B.; Allario, T.; Dambier, D.; Ollitrault, P.; Morillon, R. Tetraploid citrus rootstocks are more tolerant to salt stress than diploid. C. R. Biol. 2008. 331, 703-710.

26. Xiao, X.; Yang, F.; Zhang, S.; Korpelainen, H.; Li, C. Physiological and proteomic responses of two contrasting Populus cathayana populations to drought stress. Physiol. Plant. 2009, 136, 150-168.

27. Verslues, P.E.; Agarwal, M.; Katiyar-Agarwal, S.; Zhu, J.; Zhu, J.K. Methods and concepts in quantifying resistance to drought, salt and freezing, abiotic stresses that affect plant water status. Plant J. 2006, 45, 523-539.

28. Apel. K.; Hirt, H. Reactive oxygen species: Metabolism, oxidative stress, and signal transduction. Annu. Rev. Plant Biol. 2004, 55, 373-399.

29. Tuna, A.L.; Kaya, C.; Dikilitas, M.; Higgs, D. The combined effects of gibberellid acid and salinity on some antioxidant enzyme activities, plant growth parameters and nutritional status in maize plants. Environ. Exp. Bot. 2008, 62, 1-9.

30. Ashraf, M. Biotechnological approach of improving plant salt tolerance using antioxidants as markers. Biotechnol. Adv. 2009, 27, 84-93.

31. Barhoumi, Z.; Djebali, W.; Chaïbi, W.; Abdelly, C.; Smaoui, A. Salt impact on photosynthesis and leaf ultrastructure of Aeluropus littoralis. J. Plant Res. 2007, 120, 529-537.

32. Zapata, J.M.; Guéra, A.; Esteban-Carrasco, A.; Martín, M.; Sabater, B. Chloroplasts regulate leaf senescence: Delayed senescence in transgenic $n d h F$-defective tobacco. Cell Death Differ. 2005, 12, 1277-1284.

33. Ishikawa, T.; Yoshimura, K.; Tamoi, M.; Takeda, T.; Shigeoka, S. Alternative mRNA splicing of 3 '-terminal exons generates ascorbate peroxidase isoenzymes in spinach (Spinacia oleracea) chloroplasts. Biochem. J. 1997, 328, 795-800.

34. Ndimba, B.K.; Chivasa, S.; Simon, W.J.; Slabas, A.R. Identification of Arabidopsis salt and osmotic stress responsive proteins using two-dimensional difference gel electrophoresis and mass spectrometry. Proteomics 2005, 5, 4185-4196.

35. Portis, J.A.R. Rubisco activase: Rubisco's catalytic chaperone. Photosynth. Res. 2003, 751, 11-27.

36. Parker, R.; Flowers, T.J.; Moore, A.L.; Harpham, N.V.J. An accurate and reproducible method for proteome profiling of the effects of salt stress in the rice leaf lamina. J. Exp. Bot. 2006, 57, 1109-1118.

37. Sudhir, P.R.; Pogoryelov, D.; Kovacs, L.; Garab, G.; Murthy, S.D. The effects of salt stress on photosynthetic electron transport and thylakoid membrane proteins in the cyanobacterium Spirulina platensis. J. Biochem. Mol. Biol. 2005, 38, 481-485.

38. Ma, H.; Song, L.; Shu, Y.; Wang, S.; Niu, J.; Wang, Z.; Yu, T.; Gu, W.; Ma, H.; Baker, N.R. Comparative proteomic analysis of seedling leaves of different salt tolerant soybean genotypes. J. Proteomics 2012, 75, 1529-1546. 
39. Davletova, S.; Rizhsky, L.; Liang, H.; Sheng, A.; Oliver, D.J.; Coutu, J.; Shulaev, V.; Schlauch, K.; Mittler, R. Cytosolic ascorbate peroxidase 1 is a central component of the reactive oxygen gene network of Arabidopsisi. Plant Cell 2005, 17, 268-281.

40. Shakirova, F.M.; Bezrukova, M.V.; Khairullin, R.M. The increase in lectin level in wheat shoots under the action of salt stress. Izv. Russ. Acad. Sci. 1993, 1, 142-145.

41. Kosová, K.; Vítámvás, P.; Prášil, L.T.; Renaut, J. Plant proteome changes under abiotic stress-contribution of proteomics studies to understanding plant stress response. J. Proteomics 2011, 74, 1301-1322.

42. Wolf, B. A comprehensive system of leaf analyses and its use fore diagnosing crop nutrient status. Commun. Soil Sci. Plant Anal. 1982, 13, 1035-1059.

43. Chen, S.; Li, J.; Wang, S.; Hüttermann, A.; Altman, A. Salt, nutrient uptake and transport, and ABA of Populus euphratica; a hybrid in response to increasing soil NaCl. Trees 2001, 15, 186-194.

44. Roth, E.F.; Gilbert, J.H.S. Pyrogallol assay for SOD: Absence of a glutathione artifact. Anal. Biochem. 1984, 137, 50-53.

45. Nickel, R.S.; Cunningham, B.A. Improved peroxidase assay method using leuco 2,3,6-trichloroindophenol and application to comparative measurements of peroxidase catalysis. Anal. Biochem. 1969, 27, 292-299.

46. Nordhoff, A.; Bucheler, U.S.; Werner, D.; Schirmer, R.H. Folding of the four domains and dimerization are impaired by the Gly446 $\rightarrow$ Glu exchange in human glutathione reductase. implications for the design of antiparasitic drugs. Biochemistry 1993, 32, 4060-4066.

47. Nakano, Y.; Asad, K. Hydrogen peroxide is scavenged by ascorbate-specific peroxidase in spinach chloroplast. Plant Cell Physiol. 1981, 22, 867-880.

48. Hurkman, W.J.; Tanaka, C.K. Solubilization of plant membrane proteins for analysis by two-dimensional gel electrophoresis. Plant Physiol. 1986, 81, 802-806.

49. Bradford, M. A rapid and sensitive method for the quantification of microgram quantities of protein utilizing the principle of protein-due binding. Ann. Biochem. 1976, 72, 248-254.

(C) 2013 by the authors; licensee MDPI, Basel, Switzerland. This article is an open access article distributed under the terms and conditions of the Creative Commons Attribution license (http://creativecommons.org/licenses/by/3.0/). 